TRANSACTIONS OF THE AMERICAN MATHEMATICAL SOCIETY

Volume 348, Number 10, October 1996

\title{
MURNAGHAN-NAKAYAMA RULES FOR CHARACTERS OF IWAHORI-HECKE ALGEBRAS OF CLASSICAL TYPE
}

\author{
TOM HALVERSON AND ARUN RAM
}

\begin{abstract}
In this paper we give Murnaghan-Nakayama type formulas for computing the irreducible characters of the Iwahori-Hecke algebras of types $A_{n-1}, B_{n}$, and $D_{n}$. Our method is a generalization of a derivation of the Murnaghan-Nakayama formula for the irreducible characters of the symmetric group given by Curtis Greene. Greene's approach is to sum up the diagonal entries of the matrices of certain cycle permutations in Young's seminormal representations. The analogues of the Young seminormal representations for the Iwahori-Hecke algebras of types $A_{n-1}, B_{n}$, and $D_{n}$ were given by Hoefsmit.
\end{abstract}

\section{INTRODUCTION}

A Murnaghan-Nakayama formula for the irreducible characters of the IwahoriHecke algebras of type $A_{n-1}$ was originally found in $[\mathrm{R}]$ and in the sequence of papers $[\mathrm{KW}],[\mathrm{vdJ}]$. This formula is an analogue of the Murnaghan-Nakayama formula for computing the irreducible characters of the symmetric group. There are also analogues of the Murnaghan-Nakayama formula for computing the irreducible characters of the hyperoctahedral groups (the Weyl groups of type $B_{n}$ ) and, more generally, for any of the wreath products $\mathbb{Z}_{r}\left\{S_{n}\right.$. The formula for the hyperoctahedral group is "well known" and may even be in the works of Young [Y], but there is a very nice derivation given by J. Stembridge in [Ste]. The more general formula, in the case of $\mathbb{Z}_{r} 2 S_{n}$, can be found in [AK]. In view of these results, it is desirable to find analogous formulas for the characters of the Iwahori-Hecke algebras corresponding to Weyl groups of types $B_{n}$ and $D_{n}$.

In all of the original derivations $[\mathrm{R}],[\mathrm{KW}],[\mathrm{vdJ}]$ of the Murnaghan-Nakayama rules for Iwahori-Hecke algebras of types $A_{n-1}$ the key was essentially to use the theory symmetric functions and Schur polynomials, and the Schur-Weyl duality between the Iwahori-Hecke algebras of type $A_{n-1}$ and the Drinfel'd-Jimbo quantum groups $U_{q}(\mathfrak{g l}(m))$. This approach seems to be quite challenging for type $B_{n}$, although some progress has been made (see $[\mathrm{ATY}]$ ).

An alternate approach is to sum up the diagonal entries of the matrices in the irreducible representations. Fortunately, Hoefsmit $[\mathrm{H}]$ has given explicit analogues of the Young seminormal form of the irreducible representations of the symmetric group in the cases of the Iwahori-Hecke algebras of types $A_{n-1}, B_{n}$ and $D_{n}$. The papers [CK1] and [CK2] were able to use these explicit representations and compute the appropriate sum of diagonal elements to recover the Murnaghan-Nakayama

Received by the editors October 20, 1994.

1991 Mathematics Subject Classification. Primary 20C05; Secondary 05E05.

Supported in part by a National Science Foundation postdoctoral fellowship DMS-9107863.

(C)1996 American Mathematical Society 
rules for Iwahori-Hecke algebras of types $A_{n-1}$. (There are also several other important results in [CK1] and [CK2].)

It is, however, a nontrivial matter to do the appropriate sums in the cases of types $B_{n}$ and $D_{n}$. It is here that we come to the work of Curtis Greene [Gr]. Working in the case of the symmetric group, Greene takes an approach similar to that in [CK1] and [CK2] and sums up the diagonal entries of the matrices of the irreducible representations in Young's seminormal form. The crucial thing is that he has a beautiful way of computing this sum by using the Möbius function of a poset that is determined by the partition which indexes the irreducible representation. In fact, he has generalized the summing procedure involved in obtaining the MurnaghanNakayama formula for the symmetric group to the case of an arbitrary planar poset.

By modifying the poset result of Greene $[\mathrm{Gr}]$ to suit our needs, we are able to give a consistent method of deriving Murnaghan-Nakayama rules for the characters of the Iwahori-Hecke algebras of types $A_{n-1}, B_{n}$ and $D_{n}$.

Summary. In each case, type $A_{n-1}, B_{n}$, and $D_{n}$, we give:

(1) A definition of the Iwahori-Hecke algebra;

(2) A description of two sets of objects which label the irreducible representations and bases of the irreducible representations respectively;

(3) A complete description of the analogues of Young's seminormal representations as given by Hoefsmit $[\mathrm{H}]$;

(4) A description of the "standard" elements on which we compute character values.

(5) A derivation of the Murnaghan-Nakayama rules for the irreducible characters. We begin with the case of type $B_{n}$ in section 2 , as it is the most general and the other cases are most easily done by reducing to this case. The type $A_{n-1}$ case, given in section 3 , easily reduces to the type $B_{n}$ case, and the type $D_{n}$ case given in section 4 is only slightly more complicated than the type $B_{n}$ case. Our results show that every type $A_{n-1}$ character is the same as a type $B_{n}$ character, and that the characters in the type $D_{n}$ case can always be written as a difference of a type $B_{n}$ character and a type $A_{n}$ character. Sections 2,3 , and 4 all follow the same general format, and all rely in some way on our version of C. Green's poset result [Gr]. This result is given in section 5 and is independent of the other sections of this paper. Our main results appear in Theorems 2.20, 2.22, 3.4, and 4.35.

Remarks on the results in this paper. (1) There is recent work of G. Pfeiffer [P] which shows that indeed there is a third approach to the Murnaghan-Nakayama formulas for the characters of the Iwahori-Hecke algebras of type $A_{n-1}$. In communications with M. Geck we have learned that G. Pfeiffer has also recently proved Murnaghan-Nakayama formulas for Iwahori-Hecke algebras of types $B_{n}$ and $D_{n}$, but that written versions of his work are not yet available.

(2) Although we have not given a separate exposition for the case of the "Hecke algebras" of the wreath product $\mathbb{Z}_{r} \nmid S_{n}$, which were defined by S. Ariki and K. Koike $[\mathrm{AK}]$, it is clear that our methods apply in exactly the same way to give Murnaghan-Nakayama formulas for computing the characters of their algebras as well.

(3) In the work of Cummins and King [CK1], [CK2], "partial traces" are used to derive the Murnaghan-Nakayama rules for Iwahori-Hecke algebras of type $A_{n-1}$. The poset method used in this paper can also be used to compute (very easily) the partial traces that arise in [CK1] and [CK2]. 
(4) One of the important steps in our work in this paper is to use certain elements $D_{k}$ in the Iwahori-Hecke algebra which satisfy the following property.

The matrix of $D_{k}$ in every irreducible representation (as defined by Hoefsmit) is diagonal with eigenvalues which are plus or minus a power of $q$.

In type $B_{n}$ these elements are given by

$$
D_{k}=T_{s_{k}} T_{s_{k-1}} \ldots T_{s_{2}} T_{s_{1}} T_{s_{2}} \ldots T_{s_{k-1}} T_{s_{k}} .
$$

To our knowledge, these elements were originally discovered by Hoefsmit in the case of Iwahori-Hecke algebras of type $B_{n}$. These elements also play an important role in the work of Ariki and Koike [AK] (they probably rediscovered them, since they use them without reference to Hoefsmit). We have chosen to call these elements Hoefsmit elements. Surprisingly, it was shown in [LR] that in type $A_{n-1}$ these elements arise in a natural way from quantum groups!

(5) Let $W$ be a Weyl group and let $T_{w}, w \in W$, denote the standard basis of the corresponding Iwahori-Hecke algebra. It is still an open problem, even in type $A_{n-1}$, to give an analogue of the Murnaghan-Nakayama which can compute the irreducible characters on an arbitrary basis element $T_{w}, w \in W$. It is a result of M. Geck and G. Pfeiffer [GP] that it is sufficient to compute the characters of elements $T_{w_{i}}$ in the Iwahori-Hecke algebra, for a set of representatives $\left\{w_{i}\right\}$ of the conjugacy classes of the Weyl group $W$, where all of the $w_{i}$ are minimal length in their conjugacy class. Their result shows that there is always an algorithm for computing the character of an arbitrary basis element $T_{w}, w \in W$, of the IwahoriHecke algebra in terms of the characters of the representative elements $T_{w_{i}}$. In practice, however, this algorithm can be very complicated, and it is still hard to compute the character of an arbitrary basis element $T_{w}$. Thus, it seems desirable to have nice formulas for computing the characters of as many of the $T_{w}, w \in W$, as possible.

In this paper we give Murnaghan-Nakayama type rules for computing the characters on all "standard elements" of the Iwahori-Hecke algebras of types $A_{n-1}, B_{n}$, and $D_{n}$. In the case $B_{n}$ these are elements $T_{\pi}$ in the Iwahori-Hecke algebra which correspond to signed permutations which, in cycle notation, are of the form

$$
\begin{aligned}
\pi= & \left(1,2, \ldots,\left|l_{1}\right|-1, l_{1}\right)\left(\left|l_{1}\right|+1,\left|l_{1}\right|+2, \ldots,\left|l_{2}\right|-1, l_{2}\right) \ldots \\
& \left(\left|l_{k-1}\right|+1, \ldots, n-1, l_{k}\right)
\end{aligned}
$$

where $l_{i}$ are positive or negative integers such that $0<\left|l_{1}\right|<\left|l_{2}\right|<\cdots<\left|l_{k}\right|=n$. In each case our set of standard elements $T_{\pi}$, certainly contains a set of representative elements $T_{w_{i}}$, where $w_{i}$ are minimal length in their conjugacy class, and, in general, it contains many more elements. Thus, not only do our results, in combination with the result of Geck and Pfeiffer, completely determine the irreducible characters on representative elements, but we show that the same MurnaghanNakayama rules are able to compute the characters of many other basis elements as well.

(6) Our results in this paper seem to indicate that an analogue Schur-Weyl duality for the case of Iwahori-Hecke algebras type $B_{n}$ (up to now unknown, see [ATY]) must be quite subtle. To be more specific, if one were to hope that the method of Schur-Weyl duality and symmetric functions for the type $B_{n}$ case is similar to that used in the type $A_{n-1}$ case, then one would require symmetric 
functions, $f_{\bar{r}}$, that satisfy (in the notation of Theorem 2.20)

$$
f_{\bar{r}} s_{\lambda}=\sum_{\mu} \Delta(\mu / \lambda)(q) s_{\mu}
$$

where $s_{\lambda}$ denotes the Schur function determined by the partition $\lambda$. If this were the case, one could solve for $f_{\bar{r}}$ by writing

$$
f_{\bar{r}}=f_{\bar{r}} s_{\varnothing}=\sum_{\mu \text { a border strip }} s_{\mu} q^{c(\mu)-1}\left(-q^{-1}\right)^{r(\mu)-1}
$$

which is essentially the same as the symmetric function for type $A_{n-1}$. However this symmetric function $f_{\bar{r}}$ cannot possibly satisfy the first condition unless the characters in the type $B_{n}$ case are exactly the same as in the type $A_{n}$ case.

\section{TYPE $B_{n}, n \geq 2$}

Definition. Let $q$ and $u$ be indeterminates. The Iwahori-Hecke algebra $H B_{n}\left(u, q^{2}\right)$ of type $B_{n}$ is the associative algebra with 1 over the field $\mathbb{C}(u, q)$ given by generators $g_{1}, g_{2}, \ldots, g_{n}$ and relations

$$
\begin{aligned}
g_{i} g_{j} & =g_{j} g_{i}, \quad|i-j|>1, \\
g_{i} g_{i+1} g_{i} & =g_{i+1} g_{i} g_{i+1}, \quad 2 \leq i \leq n-1, \\
g_{1} g_{2} g_{1} g_{2} & =g_{2} g_{1} g_{2} g_{1}, \\
g_{1}^{2} & =(u-1) g_{1}+u, \\
g_{i}^{2} & =\left(q-q^{-1}\right) g_{i}+1, \quad 2 \leq i \leq n .
\end{aligned}
$$

Remark 2.2. The usual presentation of $H B_{n}(u, q)$ uses the relation $\left(g_{i}^{\prime}\right)^{2}=$ $(q-1) g_{i}^{\prime}+q$ in place of the relation $g_{i}^{2}=\left(q-q^{-1}\right) g_{i}+1$ for $2 \leq i \leq n$. One can easily convert from the primed presentation of $H B_{n}(u, q)$ to the above presentation of $H B_{n}\left(u, q^{2}\right)$ by first replacing $q$ by $q^{2}$ and then setting $g_{i}=g_{i}^{\prime} / q$.

Double Partitions and Standard Tableaux. As in [Mac], we shall identify each partition $\alpha$ with its Ferrers diagram and say that a box $b$ in $\alpha$ is in position $(i, j)$ in $\alpha$ if $b$ is in row $i$ and column $j$ of $\alpha$. The rows and columns of $\alpha$ are labeled in the same way as for matrices.

A double partition of size $n, \lambda=(\alpha, \beta)$, is an ordered pair of partitions $\alpha$ and $\beta$ such that $|\alpha|+|\beta|=n$. If $\mu=(\gamma, \rho)$ is another double partition, we write $\mu \subseteq \lambda$ if $\gamma \subseteq \alpha$ and $\rho \subseteq \beta$. In this case, we say that $\lambda / \mu=(\alpha / \gamma, \beta / \rho)$ is a skew shape. We shall refer to double partitions and skew shapes collectively as shapes. If $\lambda$ is a shape, then $\lambda^{\alpha}$ and $\lambda^{\beta}$ shall denote the first and second elements of the $\lambda$, respectively, so that $\lambda=\left(\lambda^{\alpha}, \lambda^{\beta}\right)$.

A standard tableau $L=\left(L^{\alpha}, L^{\beta}\right)$ of shape $\lambda=(\alpha, \beta)$ is a filling of the Ferrers diagram of $\lambda$ with the numbers $1,2, \ldots, n$ such that the numbers are increasing left to right across the rows of $L^{\alpha}$ and $L^{\beta}$ and increasing down the columns of $L^{\alpha}$ and $L^{\beta}$. For any shape (or skew shape) $\lambda$, let $\mathcal{L}(\lambda)$ denote the set of standard tableaux of shape $\lambda$ and, for each standard tableau $L$, let $L(k)$ denote the box containing $k$ in $L$. For example, the left picture in Figure 2.3 is a standard tableau of shape ((332), (411)). 


\begin{tabular}{|c|c|c|}
\hline 2 & 3 & 4 \\
\hline 7 & 11 & 13 \\
\hline 8 & 12 & \multicolumn{1}{|c}{} \\
\cline { 1 - 1 } &
\end{tabular}

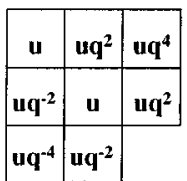

\begin{tabular}{|c|c|c|c|}
\hline 1 & 5 & 6 & 10 \\
\hline 9 & \multicolumn{2}{|l}{} \\
\cline { 1 - 1 } 14 & \multicolumn{2}{|}{} \\
\cline { 1 - 1 } & &
\end{tabular}

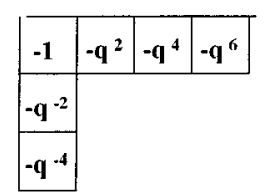

Figure 2.3

Representations. Let the content of a box $b$ of a skew shape $\lambda / \mu=\left((\lambda / \mu)^{\alpha}\right.$, $\left.(\lambda / \mu)^{\beta}\right)$ be given by

$$
c t(b)= \begin{cases}u q^{2(j-i)}, & \text { if } b \text { is in position }(i, j) \text { in }(\lambda / \mu)^{\alpha} \\ -q^{2(j-i)}, & \text { if } b \text { is in position }(i, j) \text { in }(\lambda / \mu)^{\beta} .\end{cases}
$$

For example, the contents of the boxes in the shape $((332),(411))$ are displayed in the right picture in Figure 2.3.

For each $2 \leq k \leq n$ and each standard tableau $L$ of size $n$, define

$$
\left(g_{k}\right)_{L L}=\frac{q-q^{-1}}{1-\frac{c t(L(k-1))}{c t(L(k))}} .
$$

Note that $\left(g_{k}\right)_{L L}$ depends only on the positions of the boxes containing $k$ and $k-1$ in $L$.

Let $\lambda=(\alpha, \beta)$ be a double partition of size $n$, and let

$$
V^{(\alpha, \beta)}=\mathbb{C}(u, q)-\operatorname{span}\left\{v_{L} \mid L \in \mathcal{L}(\lambda)\right\}
$$

so that the vectors $v_{L}$ form a basis of $V^{\lambda}$. Define an action of $H B_{n}\left(u, q^{2}\right)$ on $V^{\lambda}$ by defining

$$
\begin{aligned}
& g_{1} v_{L}=c t(L(1)) v_{L}, \\
& g_{i} v_{L}=\left(g_{i}\right)_{L L} v_{L}+\left(q^{-1}+\left(g_{i}\right)_{L L}\right) v_{s_{i} L}, \quad 2 \leq i \leq n,
\end{aligned}
$$

where $s_{i} L$ is the same standard tableau as $L$ except that the positions of $i$ and $i-1$ are switched in $s_{i} L$. If $s_{i} L$ is not standard, then we define $v_{s_{i} L}=0$.

Theorem 2.8 (Hoefsmit $[\mathrm{H}]$, Theorem 2.2.14). The modules $V^{(\alpha, \beta)}$, where $(\alpha, \beta)$ runs over all ordered pairs of partitions such that $|\alpha|+|\beta|=n$, form a complete set of nonisomorphic irreducible modules for $H B_{n}\left(u, q^{2}\right)$.

Hoefsmit Elements. For each $1 \leq k \leq n$ define

$$
D_{1}=g_{1}, \quad \text { and } \quad D_{k}=g_{k} g_{k-1} \ldots g_{2} g_{1} g_{2} \ldots g_{k-1} g_{k},
$$

and for each standard tableau $L$ and each entry $k$ in $L$, define

$$
\left(D_{k}\right)_{L L}=c t(L(k)) \text {. }
$$

Note that $\left(D_{k}\right)_{L L}$ depends only on the position of the box $L(k)$ and not on $k$. 
Proposition 2.11 (Hoefsmit $[\mathrm{H}]$, Proposition 3.3.3). The action of the element $D_{k}$ in the irreducible representations given by Theorem 2.8 is given by

$$
D_{k} v_{L}=\left(D_{k}\right)_{L L} v_{L}=c t(L(k)) v_{L}, \quad \text { for all standard tableaux } L .
$$

Standard Elements. Define $\overline{[n]}=\{0,1, \overline{1}, 2, \overline{2}, \ldots, n, \bar{n}\}$, and let $|\bar{i}|=|i|=i$ for each $1 \leq i \leq n$. We say that an increasing sequence in $\overline{[n]}$ is a sequence $\vec{l}=\left(l_{1}, \ldots, l_{k}\right)$ of elements of $\overline{[n]}$ such that $\left|l_{1}\right|<\cdots<\left|l_{k-1}\right|<\left|l_{k}\right|=n$.

For $1 \leq k<l \leq n$, define

$$
R_{k l}=g_{k+1} g_{k+2} \ldots g_{l}, \quad \text { and } \quad R_{k \bar{l}}=D_{k} g_{k+1} g_{k+2} \ldots g_{l},
$$

and, for each $1 \leq k \leq n$, define $R_{k k}=R_{k \bar{k}}=1$. For an increasing sequence $\vec{l}=\left(l_{1}, \ldots, l_{k}\right)$, define

$$
T_{\vec{l}}=R_{1, l_{1}} R_{\left|l_{1}\right|+1, l_{2}} \cdots R_{\left|l_{k-1}\right|+1, l_{k}} \in H B_{n}\left(u, q^{2}\right) .
$$

Remark 2.14. Let $W B_{n}$ denote the Weyl group of type $B_{n}$ with generators $s_{1}, s_{2}$, $\ldots, s_{n}$ which satisfy the Coxeter relations. For each $1 \leq k \leq n$, let $d_{k}=s_{k} s_{k-1} \ldots$ $s_{2} s_{1} s_{2} \ldots s_{k-1} s_{k}$ and, for $1 \leq k<l \leq n$, define $r_{k l}=s_{k+1} s_{k+2} \ldots s_{l}$ and $r_{k \bar{l}}=$ $d_{k} s_{k+1} s_{k+2} \ldots s_{l}$. For each $1 \leq k \leq n$, define $r_{k k}=r_{k \bar{k}}=1$. Let $\vec{l}$ be an increasing sequence in $\overline{[n]}$. Then $w_{\vec{l}}=r_{1, l_{1}} r_{\left|l_{1}\right|+1, l_{2}} \ldots r_{\left|l_{k-1}\right|+1, l_{k}}$ gives a reduced expression for the signed permutation that is given in cycle notation by

$$
\begin{aligned}
w_{\vec{l}}= & \left(1,2, \ldots,\left|l_{1}\right|-1, l_{1}\right)\left(\left|l_{1}\right|+1,\left|l_{1}\right|+2, \ldots,\left|l_{2}\right|-1, l_{2}\right) \\
& \ldots\left(\left|l_{k-1}\right|+1, \ldots,\left|l_{k}\right|-1, l_{k}\right) .
\end{aligned}
$$

Remark 2.15. M. Geck and P. Pfeiffer [GP] show that the irreducible characters of Iwahori-Hecke algebras are completely determined by computing their values on the elements $T_{w_{i}}$, where $\left\{w_{i}\right\}$ is a set of representatives of the conjugacy classse of the Weyl group $W$ such that each $w_{i}$ is minimal in its conjugacy class. This means that computing the irreducible characters on the set of standard elements $T_{\vec{l}}$, is more than sufficient to determine them.

For $1 \leq k<l \leq n$ and any standard tableau $L$ of size $n$, make the following definitions:

$$
\begin{aligned}
& \Delta_{k l}(L)=\left(g_{k+1}\right)_{L L}\left(g_{k+2}\right)_{L L} \ldots\left(g_{l}\right)_{L L}, \\
& \Delta_{k \bar{l}}(L)=\left(D_{k}\right)_{L L}\left(g_{k+1}\right)_{L L}\left(g_{k+2}\right)_{L L} \ldots\left(g_{l}\right)_{L L},
\end{aligned}
$$

and define $\Delta_{k k}(L)=\Delta_{k \bar{k}}(L)=1$, for all $1 \leq k \leq n$. Since $\left(g_{j}\right)_{L L}$ depends only on the positions if the boxes $j$ and $j-1$ in $L, \Delta_{k l}$ and $\Delta_{k \bar{l}}$ depend only on the positions of the boxes containing $k, k+1, \ldots, l$ in $L$.

Proposition 2.17. Let $\vec{l}=\left(l_{1}, \ldots, l_{k}\right)$ be an increasing sequence in $\overline{[n]}$, and let $L$ be a standard tableau of size $n$. Let $\left.T_{\vec{l}} v_{L}\right|_{v_{L}}$ denote the coefficient of $v_{L}$ in $T_{\vec{l}} v_{L}$. Then

$$
\left.T_{\vec{l}} v_{L}\right|_{v_{L}}=\Delta_{1, l_{1}}(L) \Delta_{\left|l_{1}\right|+1, l_{2}}(L) \ldots \Delta_{\left|l_{k-1}\right|+1, l_{k}}(L) .
$$

In particular, for a given sequence $\vec{l}$, the value $\left.T_{\vec{l}} v_{L}\right|_{v_{L}}$ depends only on the positions and the linear order of the boxes in $L$.

Proof. This follows from the definition of the action of $H B_{n}\left(u, q^{2}\right)$ on standard tableaux and the fact (2.7) that when $g_{i}$ acts on a standard tableau $L$ it affects only the positions of $L$ containing $i$ and $i-1$. The result follows, since $D_{j}$ acts as 
a scalar (Proposition 2.11), and $T_{\vec{l}}$ otherwise is a product (from right to left) of a decreasing sequence of generators $g_{i}$.

Characters. If $L$ is a standard tableau (of any shape, possibly of skew shape) with $n$ boxes, define

$$
\Delta(L)=\Delta_{1, n}(L) \quad \text { and } \quad \bar{\Delta}(L)=\Delta_{1, \bar{n}}(L) .
$$

For any shape $\lambda / \mu$, define

$$
\Delta(\lambda / \mu)=\sum_{L \in \mathcal{L}(\lambda / \mu)} \Delta(L) \text { and } \bar{\Delta}(\lambda / \mu)=\sum_{L \in \mathcal{L}(\lambda / \mu)} \bar{\Delta}(L) .
$$

In making these definitions, the actual values in the boxes of $L$ do not matter; only their positions and their order relative to one another are relevant. Thus, the definitions make sense when the standard tableaux have values that form a subset of $\{1,2, \ldots\}$ (with the usual linear order).

Let $\chi_{H B_{n}\left(u, q^{2}\right)}^{(\alpha, \beta)}$ denote the character of the irreducible $H B_{n}\left(u, q^{2}\right)$-representation $V^{(\alpha, \beta)}$. Recall from Remark 2.15 that it more than suffices to compute the irreducible characters on standard elements $T_{\vec{l}}$. The following theorem is our analogue of the Murnaghan-Nakayama rule.

Theorem 2.20. Let $\vec{l}$ be an increasing sequence in $\overline{[n]}$, and suppose that $\lambda=(\alpha, \beta)$ is a pair of partitions such that $|\alpha|+|\beta|=n$. Then

$$
\chi_{H B_{n}\left(u, q^{2}\right)}^{(\alpha, \beta)}\left(T_{\vec{l}}\right)=\sum_{\varnothing=\mu^{(0)} \subseteq \mu^{(1)} \subseteq \cdots \subseteq \mu^{(k)}=\lambda} \Delta\left(\mu^{(1)}\right) \Delta\left(\mu^{(2)} / \mu^{(1)}\right) \ldots \Delta\left(\mu^{(k)} / \mu^{(k-1)}\right),
$$

where the sum is over all sequences $\varnothing=\mu^{(0)} \subseteq \mu^{(1)} \subseteq \cdots \subseteq \mu^{(k)}=\lambda$ such that $\left|\mu^{(i)} / \mu^{(i-1)}\right|=\left|l_{i}\right|$, and the factor $\Delta\left(\mu^{(i)} / \mu^{(i-1)}\right)$ is barred if $l_{i}$ is barred in $\vec{l}$.

Proof. By Proposition 2.17 the character $\chi_{H B_{n}\left(u, q^{2}\right)}^{(\alpha, \beta)}$ is equal to

$$
\chi_{H B_{n}\left(u, q^{2}\right)}^{(\alpha, \beta)}\left(T_{\vec{l}}\right)=\left.\sum_{L \in \mathcal{L}(\lambda)} T_{\vec{l}} v_{L}\right|_{v_{L}}=\sum_{L \in \mathcal{L}(\lambda)} \Delta_{1, l_{1}}(L) \Delta_{\left|l_{1}\right|+1, l_{2}}(L) \ldots \Delta_{\left|l_{k-1}\right|+1, l_{k}}(L) .
$$

The result follows by collecting terms according to the positions occupied by the numbers in the various segments $\left\{1,2, \ldots,\left|l_{1}\right|\right\},\left\{\left|l_{1}\right|+1, \ldots,\left|l_{2}\right|\right\}, \ldots,\left\{\left|l_{k-1}\right|+\right.$ $\left.1, \ldots,\left|l_{k}\right|\right\}$.

In view of Theorem 2.20 it is desirable to give an explicit formula for the value of $\Delta(\lambda / \mu)$. To do so requires some further notations: The skew shape $\lambda / \mu$ is a border strip if it is connected and does not contain two boxes which are adjacent in the same northwest-to-southeast diagonal. This is equivalent to saying that $\lambda / \mu$ is connected and does not contain any $2 \times 2$ block of boxes. The skew shape $\lambda / \mu$ is a broken border strip if it does not contain any $2 \times 2$ block of boxes. Therefore, a broken border strip is a union of connected components, each of which is a border strip. Note that a double partition $(\alpha, \beta)$ with both $\alpha$ and $\beta$ nonempty has two connected components.

Drawing Ferrers diagrams as in [Mac], we say that a sharp corner in a border strip is a box with no box above it and no box to its left. A dull corner in a border strip is a box that has a box to its left and a box above it but has no box directly northwest of it. Figure 2.21 shows a broken border strip with two connected components where each of the sharp corners has been marked with an $s$ and each of the dull corners has been marked with a $\mathbf{d}$. 

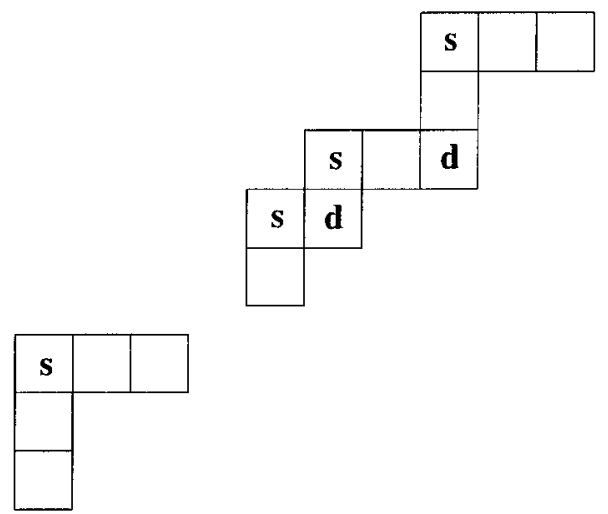

FiguRe 2.21

Theorem 2.22. Let $\lambda / \mu$ be a skew shape. Let $C C$ be the set of connected components of $\lambda / \mu$, and let $c c=|C C|$ be the number of connected components of $\lambda / \mu$. Then

$$
\Delta(\lambda / \mu)= \begin{cases}\left(q-q^{-1}\right)^{c c-1} & \prod_{b s \in C C} q^{c(b s)-1}\left(-q^{-1}\right)^{r(b s)-1} \\ 0, \quad \text { otherwise } ; & \text { if } \lambda / \mu \text { is a broken border strip } ;\end{cases}
$$

and

$$
\bar{\Delta}(\lambda / \mu)= \begin{cases}q^{c(b s)-1}\left(-q^{-1}\right)^{r(b s)-1} & \prod_{d \in D C} c t(d)^{-1} \prod_{s \in S C} c t(s) \\ & \text { if } \lambda / \mu \text { is a (connected) border strip } \\ 0, \quad \text { otherwise } ; & \end{cases}
$$

where $S C$ and $D C$ denote the sets of sharp corners and dull corners in $\lambda / \mu, r e$ spectively, and if bs is a border strip, then $r(b s)$ is the number of rows in bs, and $c(b s)$ is the number of columns in bs. The content ct(b) of a box $b$ is as given in (2.4).

Proof. Recall that

$$
\left(g_{i}\right)_{L L}=\frac{\left(q-q^{-1}\right)}{1-c t(L(i-1)) c t(L(i))^{-1}} .
$$

It then follows from the definitions of $\Delta(L)$ and $\bar{\Delta}(L)$ in (2.18), (2.16), (2.10) and (2.5) that we may apply Theorem 5.8 with $x_{b}=c t(b)$ for all boxes $b$ in $\lambda$.

Note that, for boxes $a$ and $b$,

$\frac{\left(q-q^{-1}\right)}{1-c t(a) c t(b)^{-1}}= \begin{cases}\frac{\left(q-q^{-1}\right)}{1-q^{-2}}=q, & \text { if } a \mid b, \text { i.e., } a \text { and } b \text { are adjacent in a row, } \\ \frac{\left(q-q^{-1}\right)}{1-q^{2}}=-q^{-1}, & \text { if } \frac{a}{b}, \text { i.e., } a \text { and } b \text { are adjacent in a column, }\end{cases}$ and

$\frac{1-c t(a) c t(b)^{-1}}{\left(q-q^{-1}\right)}=\frac{1-1}{q-q^{-1}}=0, \quad$ if $a / b$, i.e., $a$ and $b$ are adjacent in a diagonal. 
Thus, it follows from Theorem 5.8 that

$$
\begin{aligned}
\Delta(\lambda / \mu) & =\prod_{a \mid b} \frac{\left(q-q^{-1}\right)}{1-c t(a) c t(b)^{-1}} \prod_{a / b} \frac{\left(q-q^{-1}\right)}{1-c t(a) c t(b)^{-1}} \prod_{a / b} \frac{1-c t(a) c t(b)^{-1}}{q-q^{-1}} \\
& = \begin{cases}\left(q-q^{-1}\right)^{c c-1} \prod_{b s \in C C} q^{c(b s)-1}\left(-q^{-1}\right)^{r(b s)-1}, \\
0, & \text { otherwise, } \lambda / \mu \text { is a broken border strip; }\end{cases}
\end{aligned}
$$

and

$$
\begin{aligned}
\bar{\Delta}(\lambda / \mu)= & \prod_{d \in D C} c t(d)^{-1} \prod_{s \in S C} c t(s) \prod_{a \mid b} \frac{\left(q-q^{-1}\right)}{1-c t(a) c t(b)^{-1}} \prod_{a / b} \frac{\left(q-q^{-1}\right)}{1-c t(a) c t(b)^{-1}} \\
& \times \prod_{a / b} \frac{1-c t(a) c t(b)^{-1}}{q-q^{-1}} \\
= & \begin{cases}q^{c(b s)-1}\left(-q^{-1}\right)^{r(b s)-1} & \prod_{d \in D C} c t(d)^{-1} \prod_{s \in S C} c t(s), \\
0, & \text { otherwise. } \lambda / \mu \text { is a border strip; }\end{cases}
\end{aligned}
$$

3. Type $A_{n-1}, n \geq 2$

Definition. Let $q$ be an indeterminate. The Iwahori-Hecke algebra $H A_{n-1}\left(q^{2}\right)$ of type $A_{n-1}$ is the associative algebra with 1 over the field $\mathbb{C}(q)$ given by generators $g_{2}, \ldots, g_{n}$ (note that $g_{1}$ is missing) and relations

$$
\begin{aligned}
g_{i} g_{j} & =g_{j} g_{i}, \quad|i-j|>1, \\
g_{i} g_{i+1} g_{i} & =g_{i+1} g_{i} g_{i+1}, \quad 2 \leq i \leq n-1, \\
g_{i}^{2} & =\left(q-q^{-1}\right) g_{i}+1, \quad 2 \leq i \leq n .
\end{aligned}
$$

It is clear from the definition that the algebra $H A_{n-1}\left(q^{2}\right)$ is isomorphic to the subalgebra of $H B_{n}\left(u, q^{2}\right)$ generated by $g_{2}, \ldots, g_{n}$.

Representations. In view of the imbedding $H A_{n-1}\left(q^{2}\right) \subseteq H B_{n}\left(u, q^{2}\right)$, each of the $H B_{n}\left(q^{2}\right)$-modules $V^{(\alpha, \beta)}$ defined in section 2 is also an $H A_{n-1}\left(q^{2}\right)$-module, by restriction. For each partition $\lambda$ of $n$, let $V^{\lambda}$ be the $H A_{n-1}$-module given by

$$
V^{\lambda}=V^{(\lambda, \varnothing)} \text {. }
$$

Then we have the following theorem.

Theorem 3.3 (Hoefsmit [H], Theorem 2.3.1). The $H A_{n-1}\left(q^{2}\right)$-modules $V^{\lambda}$, where $\lambda$ runs over all partitions of $n$, form a complete set of nonisomorphic irreducible modules for $H A_{n-1}\left(q^{2}\right)$.

Standard elements. Let $\vec{l}$ be an increasing sequence in $\overline{[n]}=\{0,1, \overline{1}, 2, \overline{2}, \ldots, n, \bar{n}\}$ as defined in section 2, and assume that all $l_{i}$ in $\vec{l}$ are unbarred. Then, since the reduced word for the element $T_{\vec{l}}$ defined in (2.13) does not contain $g_{1}$, it follows that $T_{\vec{l}}$ is an element of $H A_{n-1}\left(q^{2}\right) \subseteq H B_{n}\left(u, q^{2}\right)$. As remarked in 2.15, it more than suffices to compute the irreducible $H A_{n-1}\left(q^{2}\right)$-characters on the set of standard elements $T_{\vec{l}}$. 
Characters. Let $\chi_{H A_{n-1}\left(q^{2}\right)}^{\lambda}$ denote the character of the irreducible $H A_{n-1}\left(q^{2}\right)$ module $V^{\lambda}$. The next theorem follows from the observation in the previous paragraph, and the fact that $V^{\lambda} \cong V^{(\lambda, \varnothing)}$.

Theorem 3.4. Let $\vec{l}$ be an increasing sequence as defined in section 2 and assume that all $l_{i}$ in $\vec{l}$ are unbarred. Let $T_{\vec{l}}$ be as defined in (2.13). Then $T_{\vec{l}}$ is an element of $H A_{n-1}\left(q^{2}\right)$ and, for each partition $\lambda$ of $n$,

$$
\chi_{H A_{n-1}\left(q^{2}\right)}^{\lambda}\left(T_{\vec{l}}\right)=\chi_{H B_{n}\left(u, q^{2}\right)}^{(\lambda, \varnothing)}\left(T_{\vec{l}}\right),
$$

where $\chi_{H B_{n}\left(u, q^{2}\right)}^{(\lambda, \varnothing)}$ is the $H B_{n}\left(u, q^{2}\right)$-character whose values are determined by Theorem 2.20 and Theorem 2.22.

\section{TYPe $D_{n}, n \geq 4$}

Definition. Let $q$ be an indeterminate. The Iwahori-Hecke algebra $H D_{n}\left(q^{2}\right)$ of type $D_{n}$ is the associative algebra with 1 over the field $\mathbb{C}(q)$ given by generators $\tilde{g}_{1}, \tilde{g}_{2}, \ldots, \tilde{g}_{n}$ and relations

$$
\begin{aligned}
\tilde{g}_{i} \tilde{g}_{j} & =\tilde{g}_{j} \tilde{g}_{i}, \quad|i-j|>1, i, j>1, \\
\tilde{g}_{1} \tilde{g}_{j} & =\tilde{g}_{j} \tilde{g}_{1}, \quad \text { if } j \neq 3, \\
\tilde{g}_{1} \tilde{g}_{3} \tilde{g}_{1} & =\tilde{g}_{3} \tilde{g}_{1}, \tilde{g}_{3}, \\
\tilde{g}_{i} \tilde{g}_{i+1} \tilde{g}_{i} & =\tilde{g}_{i+1} \tilde{g}_{i} \tilde{g}_{i+1}, \quad 2 \leq i \leq n-1, \\
\tilde{g}_{i}^{2} & =\left(q-q^{-1}\right) \tilde{g}_{i}+1, \quad 1 \leq i \leq n .
\end{aligned}
$$

Let $H B_{n}\left(1-q^{2}\right)$ be the algebra $H B_{n}\left(u, q^{2}\right)$ defined by generators and relations in (2.1) except with $u=1$. Define

$$
\tilde{g}_{1}=g_{1} g_{2} g_{1}, \quad \text { and } \quad \tilde{g}_{i}=g_{i}, \quad 2 \leq i \leq n .
$$

Then one easily checks that with these definitions the $\tilde{g}_{i}$ satisfy the relations in (4.1). Thus the elements $\tilde{g}_{i}, 1 \leq i \leq n$, generate a subalgebra of the algebra $H B_{n}\left(1, q^{2}\right)$ which is isomorphic to the algebra $H D_{n}\left(q^{2}\right)$.

Double Partitions and Standard Tableaux. We shall use the same notation for partitions, double partitions, shapes, and tableaux as in section 2. For each standard tableau $L=\left(L^{\alpha}, L^{\beta}\right)$ of shape $(\alpha, \beta)$ define $\sigma L$ to be the standard tableau of shape $(\beta, \alpha)$ given by $\sigma L=\left(L^{\beta}, L^{\alpha}\right)$,

$$
\begin{aligned}
\sigma: \mathcal{L}(\alpha, \beta) & \rightarrow \mathcal{L}(\beta, \alpha) \\
\left(L^{\alpha}, L^{\beta}\right) & \mapsto\left(L^{\beta}, L^{\alpha}\right) .
\end{aligned}
$$

The map $\sigma$ is an involution on the set of standard tableaux whose shape is a double partition.

Representations. Let the content of a box $b$ of a skew shape $\lambda / \mu=\left((\lambda / \mu)^{\alpha},(\lambda / \mu)^{\beta}\right)$ be given by

$$
c t(b)= \begin{cases}q^{2(j-i)}, & \text { if } b \text { is in position }(i, j) \text { in }(\lambda / \mu)^{\alpha}, \\ -q^{2(j-i)}, & \text { if } b \text { is in position }(i, j) \text { in }(\lambda / \mu)^{\beta} .\end{cases}
$$

For each standard tableau $L$, define

$$
\left(\tilde{g}_{k}\right)_{L L}=\left(g_{k}\right)_{L L}=\frac{q-q^{-1}}{1-\frac{c t(L(k-1))}{c t(L(k))}} \quad \text { for } 2 \leq k \leq n, \text { and }\left(\tilde{g}_{1}\right)_{L L}=\left(\tilde{g}_{2}\right)_{L L} .
$$


Note that $\left(\tilde{g}_{k}\right)_{L L}$ depends only on the positions of the boxes containing $k$ and $k-1$ in $L$.

Let $\lambda=(\alpha, \beta)$ be a pair of partitions such that $|\alpha|+|\beta|=n$. Let

$$
V^{(\alpha, \beta)}=\mathbb{C}(q)-\operatorname{span}\left\{v_{L} \mid L \in \mathcal{L}(\alpha, \beta)\right\}
$$

so that the vectors $v_{L}$ form a basis of the module $V^{(\alpha, \beta)}$. Recall $(2.7)$ that there is an action of $H B_{n}\left(1, q^{2}\right)$ on the vector space $V^{(\alpha, \beta)}$. Restricting this action to $H D_{n}\left(q^{2}\right)$ on $V^{\lambda}$ gives

$$
\begin{aligned}
\tilde{g}_{1} v_{L}=g_{1} g_{2} g_{1} v_{L} & =\left(\tilde{g}_{2}\right)_{L L} v_{L}-\left(q^{-1}+\left(\tilde{g}_{2}\right)_{L L}\right) v_{s_{2} L}, \\
\tilde{g}_{i} v_{L}=g_{i} v_{L} & =\left(\tilde{g}_{i}\right)_{L L} v_{L}+\left(q^{-1}+\left(\tilde{g}_{i}\right)_{L L}\right) v_{s_{i} L}, \quad 2 \leq i \leq n,
\end{aligned}
$$

for each $L \in \mathcal{L}(\alpha, \beta)$, where, as in the case of type $B_{n}$, we define $v_{s_{i} L}=0$ if $s_{i} L$ is not standard.

Now suppose $n$ is even, and let $\alpha$ be a partition such that $2|\alpha|=n$. Define

$$
\begin{aligned}
V^{(\alpha, \alpha)^{+}} & =\mathbb{C}(q)-\operatorname{span}\left\{v_{L}+v_{\sigma L} \mid L \in \mathcal{L}(\alpha, \alpha)\right\} \subseteq V^{(\alpha, \alpha)}, \\
V^{(\alpha, \alpha)^{-}} & =\mathbb{C}(q)-\operatorname{span}\left\{v_{L}-v_{\sigma L} \mid L \in \mathcal{L}(\alpha, \alpha)\right\} \subseteq V^{(\alpha, \alpha)} .
\end{aligned}
$$

Proposition 4.8 (Hoefsmit [H], Lemmas 2.3.3 and 2.3.5). (a) For each pair of partitions $(\alpha, \beta)$ such that $|\alpha|+|\beta|=n, V^{(\alpha, \beta)}$ and $V^{(\beta, \alpha)}$ are isomorphic $H D_{n}\left(q^{2}\right)$ modules.

(b) For each partition $\alpha$ such that $2|\alpha|=n$, the subspaces $V^{(\alpha, \alpha)^{ \pm}}$are $H D_{n}\left(q^{2}\right)$ submodules of $V^{(\alpha, \alpha)}$, and

$$
V^{(\alpha, \alpha)} \cong V^{(\alpha, \alpha)^{+}} \oplus V^{(\alpha, \alpha)^{-}},
$$

as $H D_{n}\left(q^{2}\right)$-modules.

Theorem 4.9 (Hoefsmit $[\mathrm{H}]$, Theorem 2.3.9). The modules $V^{(\alpha, \beta)}$, where $(\alpha, \beta)$ runs over all unordered pairs of partitions such that $\alpha \neq \beta$ and $|\alpha|+|\beta|=n$ and, when $n$ is even, the modules $V^{(\alpha, \alpha)^{+}}$and $V^{(\alpha, \alpha)^{-}}$, where $\alpha$ runs over all partitions such that $2|\alpha|=n$, form a complete set of nonisomorphic irreducible modules for $H D_{n}\left(q^{2}\right)$.

Remark 4.10. The involution $\sigma$ on standard tableaux (4.3) is a realization of the module isomorphism between the $H D_{n}\left(q^{2}\right)$-modules $V^{(\alpha, \beta)}$ and $V^{(\beta, \alpha)}$, which, in turn, comes from the automorphism of the Dynkin diagram of type $D_{n}$.

Instead of defining $V^{(\alpha, \alpha)^{ \pm}}$as in (4.7), let us define them as the quotient spaces

$$
V^{(\alpha, \alpha)^{+}}=\frac{V^{(\alpha, \alpha)}}{\left\langle v_{L}=v_{\sigma L}\right\rangle} \quad \text { and } \quad V^{(\alpha, \alpha)^{-}}=\frac{V^{(\alpha, \alpha)}}{\left\langle v_{L}=-v_{\sigma L}\right\rangle},
$$

where $\sigma$ is the involution given in (4.6) and $\left\langle v_{L}=v_{\sigma L}\right\rangle$ and $\left\langle v_{L}=-v_{\sigma L}\right\rangle$ denote the subspaces spanned by the vectors $v_{L}-v_{\sigma L}$ and by $v_{L}+v_{\sigma L}$ respectively. Clearly the two definitions of the modules $V^{(\alpha, \alpha)^{ \pm}}$are equivalent, the first represents $V^{(\alpha, \alpha)^{ \pm}}$ as subspaces of $V^{(\alpha, \alpha)}$, and the second as quotient spaces of $V^{(\alpha, \alpha)}$. The only difference is that for some computations the quotient module approach is easier; one may compute the action as in the formulas in (4.6) and then apply the relations $v_{L}= \pm v_{\sigma L}$ 
For each standard tableau $L \in \mathcal{L}(\alpha, \alpha)$, let $v_{L}^{ \pm}$denote the image of the vector $v_{L}$ in the quotient spaces $V^{(\alpha, \alpha)^{ \pm}}$respectively. Define

$$
\mathcal{L}_{2}(\alpha, \alpha)=\left\{L=\left(L_{1}^{\alpha}, L_{2}^{\alpha}\right) \in \mathcal{L}(\alpha, \alpha) \mid n \in L_{2}^{\alpha}\right\} .
$$

The vectors $v_{L}^{+}, L \in \mathcal{L}_{2}(\alpha, \alpha)$, and the vectors $v_{L}^{-}, L \in \mathcal{L}_{2}(\alpha, \alpha)$, form bases of the vector spaces $V^{(\alpha, \alpha)^{+}}$and $V^{(\alpha, \alpha)^{-}}$, respectively.

Hoefsmit Elements. Define

$$
\begin{aligned}
& \widetilde{D}_{1}=1, \quad \widetilde{D}_{2}=\tilde{g}_{2} \tilde{g}_{1}, \quad \text { and } \\
& \widetilde{D}_{k}=\tilde{g}_{k} \tilde{g}_{k-1} \ldots \tilde{g}_{3} \tilde{g}_{2} \tilde{g}_{1} \tilde{g}_{3} \tilde{g}_{4} \ldots \tilde{g}_{k-1} \tilde{g}_{k}, \quad \text { for } 3 \leq k \leq n .
\end{aligned}
$$

For each standard tableau $L$ and each entry $k$ in $L$, define

$$
\left(\widetilde{D}_{k}\right)_{L L}=\operatorname{ct}(L(1)) c t(L(k)) .
$$

Proposition 4.15. The action of the element $\widetilde{D}_{k}$ in the irreducible representations given by Theorem 4.9 is given by

$$
\begin{gathered}
\widetilde{D}_{k} v_{L}=\left(\widetilde{D}_{k}\right)_{L L} v_{L}=\operatorname{ct}(L(1)) \operatorname{ct}(L(k)) v_{L}, \\
\quad \text { for all standard tableaux } L, \text { and } \\
\widetilde{D}_{k} v_{L}^{ \pm}=\left(\widetilde{D}_{k}\right)_{L L} v_{L}^{ \pm}=\operatorname{ct}(L(1)) \operatorname{ct}(L(k)) v_{L}^{ \pm}, \\
\quad \text { for all standard tableaux } L \text { of shape }(\alpha, \alpha) .
\end{gathered}
$$

Proof. Recall that $D_{k}$ are the elements of $H B_{n}\left(1, q^{2}\right)$ given by (2.9), and use the imbedding of $H D_{n}\left(q^{2}\right)$ into $H B_{n}\left(1, q^{2}\right)$. The case $k=1$ is trivial, since $c t(L(1))=$ \pm 1 . For $k=2$, observe that $\widetilde{D}_{2}=\tilde{g}_{2} \tilde{g}_{1}=g_{2} g_{1} g_{2} g_{1}=D_{2} D_{1}$. For $3 \leq k \leq n$, note that $g_{1}$ commutes with $g_{3}, g_{4}, \ldots$ in $H B_{n}\left(1, q^{2}\right)$, and thus

$$
\begin{aligned}
\widetilde{D}_{k} & =\tilde{g}_{k} \tilde{g}_{k-1} \ldots \tilde{g}_{3} \tilde{g}_{2} \tilde{g}_{1} \tilde{g}_{3} \tilde{g}_{4} \ldots \tilde{g}_{k-1} \tilde{g}_{k} \\
& =g_{k} g_{k-1} \ldots g_{3} g_{2}\left(g_{1} g_{2} g_{1}\right) g_{3} g_{4} \ldots g_{k-1} g_{k} \\
& =g_{k} g_{k-1} \ldots g_{3} g_{2} g_{1} g_{2} g_{3} g_{4} \ldots g_{k-1} g_{k} g_{1}=D_{k} D_{1} .
\end{aligned}
$$

The result now follows from the definition of the action of $H B_{n}\left(1, q^{2}\right)$ and of $H D_{n}\left(q^{2}\right)$ on irreducible modules and Proposition 2.11.

Standard Elements. Define $\overline{[n]}=\{0,1, \overline{1}, 2, \overline{2}, \ldots, n, \bar{n}\}$, and define $|\bar{i}|=|i|=i$ for each $1 \leq i \leq n$. An unmarked increasing sequence is a sequence $\vec{l}=\left(l_{1}, \ldots, l_{k}\right)$ of elements of $\overline{[n]}$ such that

(1) $0<\left|l_{1}\right|<\cdots<\left|l_{k-1}\right|<\left|l_{k}\right|=n$,

(2) there are an even number of $l_{i}$ that are barred.

For $1 \leq k<l \leq n$, make the following definitions

$$
\widetilde{R}_{k l}=\tilde{g}_{k+1} \tilde{g}_{k+2} \ldots \tilde{g}_{l}, \quad \text { and } \quad \widetilde{R}_{k \bar{l}}=\widetilde{D}_{k} \tilde{g}_{k+1} \tilde{g}_{k+2} \ldots \tilde{g}_{l},
$$

and, for each $1 \leq k \leq n$, define $R_{k k}=R_{k \bar{k}}=1$. For each unmarked increasing sequence $\vec{l}$ define

$$
\widetilde{T}_{\vec{l}}=\widetilde{R}_{1, l_{1}} \widetilde{R}_{\left|l_{1}\right|+1, l_{2}} \ldots \widetilde{R}_{\left|l_{k-1}\right|+1, l_{k}} \in H D_{n}\left(q^{2}\right) .
$$

A marked increasing sequence is a sequence $\vec{l}=\left(\check{l}_{1}, l_{2}, \ldots, l_{k}\right)$ of elements of $\overline{[n]}$ such that

(1) $0<\left|l_{1}\right|<\cdots<\left|l_{k-1}\right|<\left|l_{k}\right|=n$,

(2) there are an even number of $l_{i}$ that are barred, 
(3) $l_{1}$ is unbarred and is marked with a check.

Define

$$
\widetilde{R}_{1 \check{1}}=1, \quad \widetilde{R}_{1 \check{2}}=\tilde{g}_{1}, \quad \text { and } \quad \widetilde{R}_{1 \check{l}}=\tilde{g}_{1} \tilde{g}_{3} \tilde{g}_{4} \ldots \tilde{g}_{l}, \quad 3 \leq l \leq n .
$$

For each marked increasing sequence $\vec{l}$ define

$$
\widetilde{T}_{\vec{l}}=\widetilde{R}_{1, \check{l}_{1}} \widetilde{R}_{\left|l_{1}\right|+1, l_{2}} \ldots \widetilde{R}_{\left|l_{k-1}\right|+1, l_{k}} \in H D_{n}\left(q^{2}\right) .
$$

We shall refer to unmarked and marked increasing sequences collectively as increasing sequences.

Characters of Representations $V^{(\alpha, \beta)},|\alpha|+|\beta|=n$. For $1 \leq k<l \leq n$ and for each standard tableau $L$, make the following definitions

$$
\begin{aligned}
k l & (L)=\left(\tilde{g}_{k+1}\right)_{L L}\left(\tilde{g}_{k+2}\right)_{L L} \ldots\left(\tilde{g}_{l}\right)_{L L}, \\
k \bar{l}(L) & =\left(\widetilde{D}_{k}\right)_{L L}\left(\tilde{g}_{k+1}\right)_{L L}\left(\tilde{g}_{k+2}\right)_{L L} \ldots\left(\tilde{g}_{l}\right)_{L L}, \\
1 \check{l}(L) & =\left(\tilde{g}_{1}\right)_{L L}\left(\tilde{g}_{3}\right)_{L L}\left(\tilde{g}_{4}\right)_{L L} \ldots\left(\tilde{g}_{l}\right)_{L L},
\end{aligned}
$$

and define ${ }_{k k}(L)={ }_{k \bar{k}}(L)={ }_{1 \check{l}}(L)=1$, for all $1 \leq k \leq n$. Note that, the relations in (2.16), (2.10), (4.5), and (4.14) imply that

$$
{ }_{k l}(L)=\Delta_{k l}(L), \quad{ }_{1 \zeta}(L)=\Delta_{1 l}(L), \quad \text { and } \quad k_{k \bar{l}}(L)=c t(L(1)) \Delta_{k \bar{l}}(L),
$$

where $\Delta_{k l}(L)$ and $\Delta_{k \bar{l}}(L)$ are the elements defined in (2.16).

Lemma 4.20. Let $\vec{l}=\left(l_{1}, \ldots, l_{k}\right)$ be an increasing sequence and let $L$ be a standard tableau. For each increasing sequence $\vec{l}$, let $\widetilde{T}_{\vec{l}}$ be the element of $H D_{n}\left(q^{2}\right)$ determined by (4.16)-(4.17) and let $T_{\vec{l}}$ be the element of $H B_{n}\left(1, q^{2}\right)$ determined by (2.13). When $\vec{l}$ is a marked increasing sequence, we ignore the mark when constructing $T_{\vec{l}}$. Let $\left.\widetilde{T}_{\vec{l}} v_{L}\right|_{v_{L}}$ denote the coefficient of $v_{L}$ in $\widetilde{T}_{\vec{l}} v_{L}$. Then

$$
\left.\widetilde{T}_{\vec{l}} v_{L}\right|_{v_{L}}=\left.T_{\vec{l}} v_{L}\right|_{v_{L}}
$$

where the right-hand side of the equality is determined by Proposition 2.1\%.

Proof. Let $\vec{l}$ be an unmarked increasing sequence and let $b$ be the number of $l_{i}$ in $\vec{l}$ that are barred. Note that the content $c t(L(1))$ is always \pm 1 and that $b$ is even. Then, it follows from (4.19) that

$$
\begin{aligned}
\widetilde{T}_{\vec{l}} u_{L} & ={ }_{1, l_{1}}(L)\left|l_{1}\right|+1, l_{2}(L) \ldots\left|l_{k-1}\right|+1, l_{k} \\
& =\operatorname{ct}(L(1))^{b} \Delta_{1, l_{1}}(L) \Delta_{\left|l_{1}\right|+1, l_{2}}(L) \ldots \Delta_{\left|l_{k-1}\right|+1, l_{k}}(L) \\
& =\Delta_{1, l_{1}}(L) \Delta_{\left|l_{1}\right|+1, l_{2}}(L) \ldots \Delta_{\left|l_{k-1}\right|+1, l_{k}}(L) \\
& =\left.T_{\vec{l}} v_{L}\right|_{v_{L}} .
\end{aligned}
$$

If $\vec{l}$ is a marked increasing sequence, then we have that

$$
\begin{aligned}
\left.\widetilde{T}_{\vec{l}} v_{L}\right|_{v_{L}} & ={ }_{1, \check{l}_{1}}(L)\left|l_{1}\right|+1, l_{2}(L) \ldots\left|l_{k-1}\right|+1, l_{k}(L) \\
& =\Delta_{1, l_{1}}(L)\left|l_{1}\right|+1, l_{2}(L) \ldots\left|l_{k-1}\right|+1, l_{k}(L) \\
& =\left.T_{\vec{l}} v_{L}\right|_{v_{L}},
\end{aligned}
$$

where the last equality follows exactly as in the unmarked case. 
Theorem 4.21. Let $\chi_{H D_{n}\left(q^{2}\right)}^{(\alpha, \beta)}$ denote the characters of the $H D_{n}\left(q^{2}\right)$-modules $V^{(\alpha, \beta)},|\alpha|+|\beta|=n$. For each increasing sequence $\vec{l}$, let $\widetilde{T}_{\vec{l}}$ be the element of $H D_{n}\left(q^{2}\right)$ determined by (4.16)-(4.17), and let $T_{\vec{l}}$ be the element of $H B_{n}\left(1, q^{2}\right)$ determined by (2.13). When $\vec{l}$ is a marked increasing sequence, we ignore the mark when constructing $T_{\vec{l}}$. Then

$$
\chi_{H D_{n}\left(q^{2}\right)}^{(\alpha, \beta)}\left(\widetilde{T}_{\vec{l}}\right)=\chi_{H B_{n}\left(1, q^{2}\right)}^{(\alpha, \beta)}\left(T_{\vec{l}}\right),
$$

where $\chi_{H B_{n}\left(1, q^{2}\right)}^{(\alpha, \beta)}$ are the irreducible characters of $H B_{n}\left(1, q^{2}\right)$ as determined by Theorems 2.20 and 2.22.

Proof. The character value $\chi_{H D_{n}\left(q^{2}\right)}^{(\alpha, \beta)}\left(\widetilde{T}_{\vec{l}}\right)$ is equal to

$$
\chi_{H D_{n}\left(q^{2}\right)}^{(\alpha, \beta)}\left(\widetilde{T}_{\vec{l}}\right)=\left.\sum_{L \in \mathcal{L}(\alpha, \beta)} \widetilde{T}_{\vec{l}} v_{L}\right|_{v_{L}}=\left.\sum_{L \in \mathcal{L}(\alpha, \beta)} T_{\vec{l}} v_{L}\right|_{v_{L}}=\chi_{H B_{n}\left(1, q^{2}\right)}^{(\alpha, \beta)}\left(T_{\vec{l}}\right),
$$

by Lemma 4.20 .

Characters of Representations $V^{(\alpha, \alpha)^{ \pm}}, 2|\alpha|=n$. We shall say that an increasing sequence $\vec{l}=\left(l_{1}, \ldots, l_{k}\right)$ or $\vec{l}=\left(\breve{l}_{1}, \ldots, l_{k}\right)$ is even if $\left|l_{i}\right|$ is even for all $1 \leq i \leq k$. If $\vec{l}$ is even, then (ignoring the factors of the form $\widetilde{D}_{j}$ ) each $\tilde{g}_{2 k}, k>1$, appears as a factor in the reduced expression for $\widetilde{T}_{\vec{l}}$, and either $\tilde{g}_{1}$ or $\tilde{g}_{2}$ appears as a factor in $\widetilde{T}_{\vec{l}}$.

A standard tableau $L=\left(L_{1}^{\alpha}, L_{2}^{\alpha}\right) \in \mathcal{L}(\alpha, \alpha)$ is alternating if, for each $1 \leq k \leq$ $n / 2$, the boxes of $L$ containing the values $2 k-1$ and $2 k$ are in the same position in the two subtableaux $L_{1}^{\alpha}$ and $L_{2}^{\alpha}$; see Figure 4.22 .

is an alternating standard tableau of shape $((421),(421))$. Recall the definition of $\mathcal{L}_{2}(\alpha, \alpha)$ from $(4.12)$, and let $\mathcal{A L}_{2}(\alpha, \alpha)$ denote the set of alternating standard tableaux $L \in \mathcal{L}_{2}(\alpha, \alpha)$.

Recall from section 2 that if $L$ is a standard tableau, then $s_{i} L$ is the same tableau as $L$ except that the positions of $i$ and $i-1$ are switched in $s_{i} L$. For each $1 \leq k \leq n / 2$ define $e_{2 k} L=s_{2 k} s_{2 k+2} \ldots s_{n-2} s_{n} L$. Note that if $L$ is an alternating standard tableau, then $e_{2} L=\sigma L$, where $\sigma$ is the involution of $\mathcal{L}(\alpha, \alpha)$ given in (4.3). In accordance with the definitions (4.5) (which are supposed to be somewhat
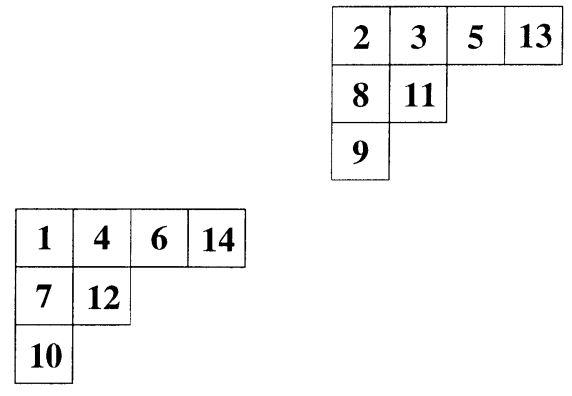

FiguRe 4.22 
reminiscent of matrix notation), we set

$$
\begin{aligned}
\left(\tilde{g}_{2 k}\right)_{e_{2 k} L, e_{2 k+2} L} & =\left(q^{-1}+\left(\tilde{g}_{2 k}\right)_{e_{2 k+2} L, e_{2 k+2} L}\right), \quad \text { for } 1 \leq k \leq n / 2, \\
\left(\tilde{g}_{1}\right)_{e_{2} L, e_{4} L} & =-\left(q^{-1}+\left(\tilde{g}_{2}\right)_{e_{4} L, e_{4} L}\right),
\end{aligned}
$$

for each standard tableau $L$. For each standard tableau $L$, define

$$
\begin{aligned}
\mathcal{O}_{1,2 l}(L)= & \mathcal{O}_{1, \overline{2 l}}(L) \\
= & \left(\tilde{g}_{2}\right)_{e_{2} L, e_{4} L}\left(\tilde{g}_{3}\right)_{e_{4} L, e_{4} L}\left(\tilde{g}_{4}\right)_{e_{4} L, e_{6} L}\left(\tilde{g}_{5}\right)_{e_{6} L, e_{6} L} \cdots \\
& \left(\tilde{g}_{2 l-1}\right)_{e_{2 l} L, e_{2 l} L}\left(\tilde{g}_{2 l}\right)_{e_{2 l} L, e_{2 l+2} L}, \\
\mathcal{O}_{1, \check{2}_{l} l}(L)= & \left(\tilde{g}_{1}\right)_{e_{2} L, e_{4} L}\left(\tilde{g}_{3}\right)_{e_{4} L, e_{4} L}\left(g_{4}\right)_{e_{4} L, e_{6} L}\left(\tilde{g}_{5}\right)_{e_{6} L, e_{6} L} \cdots \\
& \left(\tilde{g}_{2 l-1}\right)_{e_{2 l} L, e_{2 l} L}\left(\tilde{g}_{2 l}\right)_{e_{2 l} L, e_{2 l+2} L},
\end{aligned}
$$

and define

$$
\begin{aligned}
\mathcal{O}_{2 k+1,2 l}(L)= & \left(\tilde{g}_{2 k+2}\right)_{e_{2 k+2} L, e_{2 k+4} L}\left(\tilde{g}_{2 k+3}\right)_{e_{2 k+4} L, e_{2 k+4} L}\left(\tilde{g}_{2 k+4}\right)_{e_{2 k+4} L, e_{2 k+6} L} \\
& \cdot\left(\tilde{g}_{2 k+5}\right)_{e_{2 k+6} L, e_{2 k+6} L} \ldots\left(\tilde{g}_{2 l-1}\right)_{e_{2 l} L, e_{2 l} L}\left(\tilde{g}_{2 l}\right)_{e_{2 l} L, e_{2 l+2} L}, \text { and } \\
\mathcal{O}_{2 k+1, \overline{2 l}}= & \left(\widetilde{D}_{2 k+1}\right)_{e_{2 k+2} L, e_{2 k+2} L} \mathcal{O}_{2 k+1,2 l}(L), \quad \text { for } k>0 .
\end{aligned}
$$

Notice that $\left(\tilde{g}_{1}\right)_{e_{2} L, e_{4} L}=-\left(\tilde{g}_{2}\right)_{e_{2} L, e_{4} L}$, so

$$
\mathcal{O}_{1,2 l}(L)=\mathcal{O}_{1, \overline{2 l}}(L)=-\mathcal{O}_{1, \check{2} l}(L) .
$$

If $\vec{l}$ is an even increasing sequence, and $L$ is an alternating standard tableau, define

$$
\mathcal{O}_{\bar{l}}(L)=\mathcal{O}_{1, l_{1}}(L) \mathcal{O}_{\left|l_{1}\right|+1, l_{2}}(L) \ldots \mathcal{O}_{\left|l_{k-1}\right|+1, l_{k}}(L) .
$$

Lemma 4.26. Let $\vec{l}=\left(l_{1}, \ldots, l_{k}\right)$ be an increasing sequence, and let $L$ be a standard tableau. Let $\widetilde{T}_{\vec{l}}$ be the element of $H D_{n}\left(q^{2}\right)$ determined by (4.16)-(4.17) and let $T_{\vec{l}}$ be the element of $H B_{n}\left(1, q^{2}\right)$ determined by (2.13). When $\vec{l}$ is a marked increasing sequence, we ignore the mark when constructing $T_{\vec{l}}$. Let $\left.\widetilde{T}_{\vec{l}} v_{L}\right|_{v_{L}}$ denote the coefficient of $v_{L}$ in $\widetilde{T}_{\vec{l}} v_{L}$. Then

$$
\left.\widetilde{T}_{\vec{l}} v_{L}^{ \pm}\right|_{v_{L}^{ \pm}}= \begin{cases}\left.T_{\vec{l}} v_{L}\right|_{v_{L}} \pm \mathcal{O}_{\vec{l}}(L), & \text { if } \vec{l} \text { is even, and } L \text { is alternating; } \\ \left.T_{\vec{l}} v_{L}\right|_{v_{L}}, & \text { otherwise. }\end{cases}
$$

Proof. Suppose that $L$ is an alternating standard tableau. Then $e_{2} L=\sigma L$, where $\sigma$ is the involution given in (4.3). Then, in $V^{(\alpha, \alpha)}$,

$$
\widetilde{T}_{\vec{l}} v_{L}={ }_{\vec{l}}(L) v_{L}+\mathcal{O}_{\vec{l}}(L) v_{\sigma L}+\left\{\begin{array}{c}
\text { other terms } \\
\text { not containing } \\
v_{L} \text { or } v_{\sigma L}
\end{array}\right\} .
$$

It follows that, in the quotient spaces $V^{(\alpha, \alpha)^{ \pm}}$, we have

$$
\widetilde{T}_{\vec{l}} v_{L}^{ \pm}=\left({ }_{\vec{l}}(L) \pm \mathcal{O}_{\vec{l}}(L)\right) v_{L}^{ \pm}+\left\{\begin{array}{c}
\text { other terms } \\
\text { not containing } \\
v_{L}^{ \pm}
\end{array}\right\}
$$

since $v_{L}^{ \pm}= \pm v_{\sigma L}^{ \pm}$in $V^{(\alpha, \alpha)^{ \pm}}$. The lemma now follows exactly as in the proof of Lemma 4.20 . 
Proposition 4.27. Let $\vec{l}$ be an increasing sequence, and let $\widetilde{T}_{\vec{l}}$ be the element of $H D_{n}\left(q^{2}\right)$ determined by (4.16)-(4.17) and let $T_{\vec{l}}$ be the element of $H B_{n}\left(1, q^{2}\right)$ determined by (2.13). When $\vec{l}$ is a marked increasing sequence, we ignore the mark when constructing $T_{\vec{l}}$. Then

$$
\chi_{H D_{n}\left(q^{2}\right)}^{(\alpha, \alpha)^{ \pm}}\left(\widetilde{T}_{\vec{l}}\right)=\left\{\begin{array}{l}
\frac{1}{2} \chi_{H B_{n}\left(1, q^{2}\right)}^{(\alpha, \alpha)}\left(T_{\vec{l}}\right) \pm \sum_{L \in \mathcal{A} \mathcal{L}_{2}(\alpha, \alpha)} \mathcal{O}_{\vec{l}}(L), \quad \text { if } \vec{l} \text { is even }, \\
\frac{1}{2} \chi_{H B_{n}\left(1, q^{2}\right)}^{(\alpha, \alpha)}\left(T_{\vec{l}}\right), \quad \text { otherwise, }
\end{array}\right.
$$

where $\chi_{H D_{n}\left(q^{2}\right)}^{(\alpha, \alpha)^{ \pm}}$is the irreducible $H D_{n}\left(q^{2}\right)$-character corresponding to $V^{(\alpha, \alpha)^{ \pm}}$.

Proof. Let us make the following notation:

$$
\begin{aligned}
& \mathcal{L}_{1}(\alpha, \alpha)=\left\{L=\left(L_{1}^{\alpha}, L_{2}^{\alpha}\right) \in \mathcal{L}(\alpha, \alpha) \mid n \in L_{1}^{\alpha}\right\}, \\
& \mathcal{L}_{2}(\alpha, \alpha)=\left\{L=\left(L_{1}^{\alpha}, L_{2}^{\alpha}\right) \in \mathcal{L}(\alpha, \alpha) \mid n \in L_{2}^{\alpha}\right\} .
\end{aligned}
$$

Assume that $\vec{l}$ is even. Then it follows from Lemma 4.26 that

$$
\chi_{H D_{n}\left(q^{2}\right)}^{(\alpha, \alpha)^{ \pm}}\left(\widetilde{T}_{\vec{l}}\right)=\left.\sum_{L \in \mathcal{L}_{2}(\alpha, \alpha)} T_{\vec{l}} v_{L}\right|_{v_{L}} \pm \sum_{L \in \mathcal{A} \mathcal{L}_{2}(\alpha, \alpha)} \mathcal{O}_{\vec{l}}(L) .
$$

Thus,

$$
\chi_{H D_{n}\left(q^{2}\right)}^{(\alpha, \alpha)^{+}}\left(\widetilde{T}_{\vec{l}}\right)+\chi_{H D_{n}\left(q^{2}\right)}^{(\alpha, \alpha)^{-}}\left(\widetilde{T}_{\vec{l}}\right)=\left.2 \sum_{L \in \mathcal{L}_{2}(\alpha, \alpha)} T_{\vec{l}} v_{L}\right|_{v_{L}}
$$

By using Theorem 4.21 and Proposition 4.8(b),

$$
\chi_{H B_{n}\left(1, q^{2}\right)}^{(\alpha, \alpha)}\left(T_{\vec{l}}\right)=\chi_{H D_{n}\left(q^{2}\right)}^{(\alpha, \alpha)}\left(\widetilde{T}_{\vec{l}}\right)=\chi_{H D_{n}\left(q^{2}\right)}^{(\alpha, \alpha)^{+}}\left(\widetilde{T}_{\vec{l}}\right)+\chi_{H D_{n}\left(q^{2}\right)}^{(\alpha, \alpha)^{-}}\left(\widetilde{T}_{\vec{l}}\right) .
$$

Thus,

$$
\left.\sum_{L \in \mathcal{L}_{2}(\alpha, \alpha)} T_{\vec{l}} v_{L}\right|_{v_{L}}=\frac{1}{2} \chi_{H B_{n}\left(1, q^{2}\right)}^{(\alpha, \alpha)}\left(T_{\vec{l}}\right)
$$

and the result follows. The proof for the case when $\vec{l}$ is not even is exactly the same except that the term $\pm \sum \mathcal{O}_{\vec{l}}(L)$ does not appear.

Let $\alpha$ be a partition of $n / 2$. A signed standard tableau of shape $\alpha$ is a standard tableau of shape $\alpha$ such that each box of $\alpha$ also has associated with it a sign + or -. Let $\mathcal{S} \mathcal{T}_{\varepsilon}(\alpha)$ denote the set of signed standard tableaux $L_{\varepsilon}^{\alpha}$ of shape $\alpha$ such that the product of the signs is always $(-1)^{n / 2-1}$. Recall that $\mathcal{A L}_{2}(\alpha, \alpha)$ is the set of alternating standard tableaux $L \in \mathcal{L}_{2}(\alpha, \alpha)$. Define a bijection

$$
\begin{aligned}
\varphi: \mathcal{A L}_{2}(\alpha, \alpha) & \rightarrow \mathcal{S} \mathcal{T}_{\varepsilon}(\alpha) \\
L & \mapsto L_{\varepsilon}^{\alpha}
\end{aligned}
$$

as follows. Let $L=\left(L_{1}^{\alpha}, L_{2}^{\alpha}\right)$ be an alternating standard tableau, and let $L_{\varepsilon}^{\alpha}=\varphi(L)$ be the standard tableaux such that $k$ has the same position in $L_{\varepsilon}^{\alpha}$ as $2 k-1$ and $2 k$ have in the tableaux $L_{1}^{\alpha}$ and $L_{2}^{\alpha}$. Assign the box containing $k$ of $L_{\varepsilon}^{\alpha}$ a + or depending on whether or not $2 k-1$ and $2 k-2$ are in the same tableau $L_{i}^{\alpha}$. Give the box containing 1 the same sign as the content $c t(L(1))$ of the box containing 1 in $L$. Figure 4.30 illustrates the bijection $\varphi$.

Let $\varepsilon\left(L_{\varepsilon}^{\alpha}(k)\right)$ denote the sign of the box containing $k$ in $L_{\varepsilon}^{\alpha}$, and let $\operatorname{ct}\left(L_{\varepsilon}^{\alpha}(k)\right)$ denote the content of the box containing $k$ in $L_{\varepsilon}^{\alpha}$ as given by (4.4). 


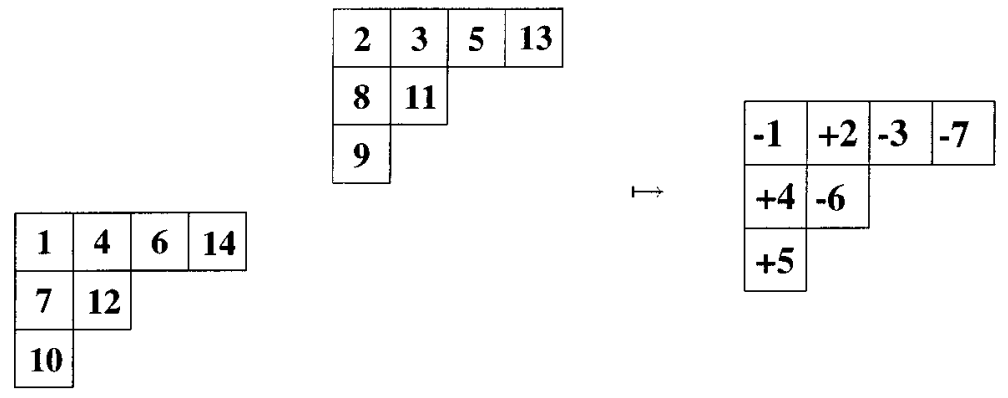

FiguRE 4.30

Proposition 4.31. Let $\alpha$ be a partition such that $2|\alpha|=n$. Let $L=\left(L_{1}^{\alpha}, L_{2}^{\alpha}\right)$ be an alternating standard tableau of shape $(\alpha, \alpha)$ and let $L_{\varepsilon}^{\alpha}=\varphi(L)$. Let $\mathcal{O}_{1,2 l}(L)$, $\mathcal{O}_{1, \check{2 l}}(L), \mathcal{O}_{2 k+1,2 l}(L)$ and $\mathcal{O}_{2 k+1, \overline{2 l}}(L)$ be as defined above (4.24), and define

$$
\begin{array}{ll}
\mathcal{O}_{1, l}\left(L_{\varepsilon}^{\alpha}\right)=\mathcal{O}_{1,2 l}(L), & \mathcal{O}_{k+1, l}\left(L_{\varepsilon}^{\alpha}\right)=\mathcal{O}_{2 k+1,2 l}(L), \\
\mathcal{O}_{1, \check{l}}\left(L_{\varepsilon}^{\alpha}\right)=\mathcal{O}_{1, \check{2} l}(L), & \mathcal{O}_{k+1, \bar{l}}\left(L_{\varepsilon}^{\alpha}\right)=\mathcal{O}_{2 k+1, \overline{2 l}}(L), \\
\mathcal{O}_{1, \bar{l}}\left(L_{\varepsilon}^{\alpha}\right)=\mathcal{O}_{1, \overline{2 l}}(L) . &
\end{array}
$$

Then

(a) $\mathcal{O}_{1, l}\left(L_{\varepsilon}^{\alpha}\right)=\mathcal{O}_{1, \bar{l}}\left(L_{\varepsilon}^{\alpha}\right)=-\mathcal{O}_{1, \bar{l}}\left(L_{\varepsilon}^{\alpha}\right)=\left(\frac{q+q^{-1}}{2}\right)^{l} \prod_{j=2}^{l} \frac{q-q^{-1}}{1+\frac{c t\left(L_{\varepsilon}^{\alpha}(j-1)\right)}{\varepsilon\left(L_{\varepsilon}^{\alpha}(j)\right) c t\left(L_{\varepsilon}^{\alpha}(j)\right)}}$

and, for $k>0$,

$$
\begin{aligned}
\mathcal{O}_{k+1, l}\left(L_{\varepsilon}^{\alpha}\right) & =\left(\frac{q+q^{-1}}{2}\right)^{l-k} \prod_{j=k+2}^{l} \frac{q-q^{-1}}{1+\frac{c t\left(L_{\varepsilon}^{\alpha}(j-1)\right)}{\varepsilon}\left(L_{\varepsilon}^{\alpha}(j)\right) c t\left(L_{\varepsilon}^{\alpha}(j)\right)}, \\
\mathcal{O}_{k+1, \bar{l}}\left(L_{\varepsilon}^{\alpha}\right) & =(-1)^{k+1}\left(\prod_{j=2}^{k+1} \varepsilon\left(L_{\varepsilon}^{\alpha}(j)\right)\right) \operatorname{ct}\left(L_{\varepsilon}^{\alpha}(k+1)\right) \mathcal{O}_{k+1, l}\left(L_{\varepsilon}^{\alpha}\right) .
\end{aligned}
$$

Proof. The first two equalities in (a) follow immediately from (4.24). In view of the definitions of $\mathcal{O}_{2 k+1,2 l}(L)$ and $\mathcal{O}_{2 k+1,2 l}(L)$, the remaining assertions will be proved by computing values of $\left(\tilde{g}_{2 k}\right)_{e_{2 k} L, e_{2 k+2} L},\left(\tilde{g}_{1}\right)_{e_{2} L, e_{4} L},\left(\tilde{g}_{2 k-1}\right)_{e_{2 k-1} L, e_{2 k-1} L}$, and $\left(\widetilde{D}_{2 k+1}\right)_{e_{2 k+1} L, e_{2 k+1} L}$.

Since $2 k-1$ and $2 k$ are in the same position in $e_{2 k+2} L$ as they are in $L$, we see that $\left(\tilde{g}_{2 k}\right)_{e_{2 k+2} L, e_{2 k+2} L}=\left(\tilde{g}_{2 k}\right)_{L L}$. Considering definitions (4.23) and (4.5), we have

$$
\left(\tilde{g}_{1}\right)_{e_{2} L, e_{4} L}=-\left(q^{-1}+\left(\tilde{g}_{2}\right)_{e_{4} L, e_{4} L}\right)=-\left(q^{-1}+\frac{q-q^{-1}}{1-\frac{c t(L(1))}{c t(L(2))}}\right)
$$

and

$$
\begin{array}{r}
\left(\tilde{g}_{2 k}\right)_{e_{2 k} L, e_{2 k+2} L}=q^{-1}+\left(\tilde{g}_{2 k}\right)_{e_{2 k+2} L, e_{2 k+2} L}=q^{-1}+\frac{q-q^{-1}}{1-\frac{c t(L(2 k-1))}{c t(L(2 k))}} \\
1 \leq k \leq n / 2
\end{array}
$$


Now, $c t(L(2 k-1))=-c t(L(2 k))$, since $2 k-1$ and $2 k$ are in the same position but in different tableaux of the pair $\left(L_{1}^{\alpha}, L_{2}^{\alpha}\right)$. So we have

$$
\left(\tilde{g}_{1}\right)_{e_{2} L, e_{4} L}=-\left(q^{-1}+\frac{q-q^{-1}}{1-(-1)}\right)=-\frac{q+q^{-1}}{2}
$$

and

$$
\left(\tilde{g}_{2 k}\right)_{e_{2 k} L, e_{2 k+2} L}=q^{-1}+\frac{q-q^{-1}}{1-(-1)}=\frac{q+q^{-1}}{2}, \quad 1 \leq k \leq n / 2 .
$$

For $1 \leq k \leq n / 2$, the permutation $e_{2 k}$ switches $2 k-1$ and $2 k$ in $L$, so $c t\left(e_{2 k} L(2 k-1)\right)=-c t(L(2 k-1))$, and $e_{2 k}$ fixes $2 k-2$ in $L$, so $c t\left(e_{2 k} L(2 k-2)\right)=$ $c t(L(2 k-2))$. Therefore,

$$
\frac{c t\left(e_{2 k} L(2 k-2)\right)}{c t\left(e_{2 k} L(2 k-1)\right)}=\frac{c t(L(2 k-2))}{-c t(L(2 k-1))}=\frac{c t\left(L_{\varepsilon}^{\alpha}(k-1)\right)}{-\varepsilon\left(L_{\varepsilon}^{\alpha}(k)\right) c t\left(L_{\varepsilon}^{\alpha}(k)\right)} .
$$

Thus, for $1<k \leq n / 2$,

$$
\left(\tilde{g}_{2 k-1}\right)_{e_{2 k} L, e_{2 k} L}=\frac{q-q^{-1}}{1-\frac{c t\left(e_{2 k} L(2 k-2)\right)}{c t\left(e_{2 k} L(2 k-1)\right)}}=\frac{q-q^{-1}}{1+\frac{c t\left(L_{\varepsilon}^{\alpha}(k-1)\right)}{\varepsilon\left(L_{\varepsilon}^{\alpha}(k)\right) c t\left(L_{\varepsilon}^{\alpha}(k)\right)}} .
$$

By induction (or by looking at an example), one can readily check that

$$
L(2 k+1) \text { is in } L_{1}^{\alpha} \text { if }(-1)^{k}\left(\prod_{j=1}^{k+1} \varepsilon\left(L_{\varepsilon}^{\alpha}(j)\right)\right)=1
$$

and

$$
L(2 k+1) \text { is in } L_{2}^{\alpha} \text { if }(-1)^{k}\left(\prod_{j=1}^{k+1} \varepsilon\left(L_{\varepsilon}^{\alpha}(j)\right)\right)=-1 .
$$

It follows that,

$$
\operatorname{ct}(L(2 k+1))=(-1)^{k}\left(\prod_{j=1}^{k+1} \varepsilon\left(L_{\varepsilon}^{\alpha}(j)\right)\right) \operatorname{ct}\left(L_{\varepsilon}^{\alpha}(k+1)\right) .
$$

Then, for $k>1$,

$$
\begin{aligned}
\left(\widetilde{D}_{2 k+1}\right)_{e_{2 k+1} L, e_{2 k+1} L} & =c t\left(e_{2 k+1} L(1)\right) c t\left(e_{2 k+2} L(2 k+1)\right) \\
& =c t(L(1))(-c t(L(2 k+1))) \\
& =\varepsilon\left(L_{\varepsilon}^{\alpha}(1)\right)\left(-(-1)^{k} \prod_{j=1}^{k+1} \varepsilon\left(L_{\varepsilon}^{\alpha}(j)\right)\right) \operatorname{ct}\left(L_{\varepsilon}^{\alpha}(k+1)\right) \\
& =(-1)^{k+1}\left(\prod_{j=2}^{k+1} \varepsilon\left(L_{\varepsilon}^{\alpha}(j)\right)\right) \operatorname{ct}\left(L_{\varepsilon}^{\alpha}(k+1)\right) .
\end{aligned}
$$

The remaining assertions follow from (1), (2), (3), (4) and the definitions of $\mathcal{O}_{2 k+1,2 l}(L)$ and $\mathcal{O}_{2 k, \overline{2 l}}(L)$. 
Our goal is to compute the sum of the $\mathcal{O}_{\vec{l}}(L)$ over all $L \in \mathcal{A} \mathcal{L}_{2}(\alpha, \alpha)$. Clearly, it is equivalent to sum the values $\mathcal{O}_{\vec{l}}\left(L_{\varepsilon}^{\alpha}\right)$ over all $L_{\varepsilon}^{\alpha} \in \mathcal{S} \mathcal{T}_{\varepsilon}(\alpha)$. We shall do this sum in two steps: first we sum over all possible choices of the signs in a signed standard tableau and then over all fillings.

For each standard tableau $L^{\alpha}$ (unsigned) of shape $\alpha$ define

$$
\begin{aligned}
\mathcal{O}_{1, l}\left(L^{\alpha}\right) & =\sum_{L_{\varepsilon}^{\alpha}} \mathcal{O}_{1, l}\left(L_{\varepsilon}^{\alpha}\right), \quad \mathcal{O}_{1, \bar{l}}\left(L^{\alpha}\right)=\sum_{L_{\varepsilon}^{\alpha}} \mathcal{O}_{1, \bar{l}}\left(L_{\varepsilon}^{\alpha}\right), \\
\mathcal{O}_{1, \breve{l}}\left(L^{\alpha}\right) & =\sum_{L_{\varepsilon}^{\alpha}} \mathcal{O}_{1, \breve{1}}\left(L_{\varepsilon}^{\alpha}\right), \\
\mathcal{O}_{k+1, l}\left(L^{\alpha}\right) & =\sum_{L_{\varepsilon}^{\alpha}} \mathcal{O}_{k+1, l}\left(L_{\varepsilon}^{\alpha}\right), \quad \text { and } \quad \mathcal{O}_{k+1, \bar{l}}\left(L^{\alpha}\right)=\sum_{L_{\varepsilon}^{\alpha}} \mathcal{O}_{k+1, \bar{l}}\left(L_{\varepsilon}^{\alpha}\right),
\end{aligned}
$$

where, in each case, we sum over all signed tableaux $L_{\varepsilon}^{\alpha}$ which equal $L^{\alpha}$ when the signs are ignored. This is equivalent to summing over all choices of signs for each box $L_{\varepsilon}^{\alpha}(i)$ for $1<i \leq n / 2$. The $\operatorname{sign} \varepsilon\left(L_{\varepsilon}^{\alpha}(1)\right)$ is forced by the condition that the product of the signs in $L_{\varepsilon}^{\alpha}$ is $(-1)^{n / 2-1}$, so the first three sums are slightly different than the last two. Thus, we may write

$$
\begin{aligned}
\mathcal{O}_{1, l}\left(L^{\alpha}\right) & =\sum_{\substack{\varepsilon(T(j))= \pm 1 \\
1<j \leq l}} \mathcal{O}_{1, l}\left(L_{\varepsilon}^{\alpha}\right), \quad \mathcal{O}_{1, \bar{l}}\left(L^{\alpha}\right)=\sum_{\substack{\varepsilon(T(j))= \pm 1 \\
1<j \leq l}} \mathcal{O}_{1, \bar{l}}\left(L_{\varepsilon}^{\alpha}\right), \\
\mathcal{O}_{1, \check{l}}\left(L^{\alpha}\right)= & \sum_{\substack{\varepsilon(T(j))= \pm 1 \\
1<j \leq l}} \mathcal{O}_{1, \check{l}}\left(L_{\varepsilon}^{\alpha}\right), \\
\mathcal{O}_{k+1, l}\left(L^{\alpha}\right)= & \sum_{\substack{\varepsilon(T(j))= \pm 1 \\
k+1 \leq j \leq l}} \mathcal{O}_{k+1, l}\left(L_{\varepsilon}^{\alpha}\right), \quad \text { and } \quad \mathcal{O}_{k+1, \bar{l}}\left(L^{\alpha}\right)=\sum_{\substack{\varepsilon(T(j))= \pm 1 \\
k+1 \leq j \leq l}} \mathcal{O}_{k+1, \bar{l}}\left(L_{\varepsilon}^{\alpha}\right) .
\end{aligned}
$$

Proposition 4.34. Let $L^{\alpha}$ be an (unsigned) standard tableau of shape $\alpha$. Then using the definitions of (4.32), we have

(a) $\mathcal{O}_{1, l}\left(L^{\alpha}\right)=\mathcal{O}_{1, \bar{l}}\left(L^{\alpha}\right)=-\mathcal{O}_{1, \check{l}}\left(L^{\alpha}\right)=\frac{\left(q+q^{-1}\right)}{2} \prod_{j=2}^{l} \frac{\left(q^{2}-q^{-2}\right)}{1-\frac{c t\left(L^{\alpha}(j-1)\right)^{2}}{c t\left(L^{\alpha}(j)\right)^{2}}}$,

and, for $k>0$,

$$
\mathcal{O}_{k+1, l}\left(L^{\alpha}\right)=\left(q+q^{-1}\right) \prod_{j=k+2}^{l} \frac{\left(q^{2}-q^{-2}\right)}{1-\frac{c t\left(L^{\alpha}(j-1)\right)^{2}}{c t\left(L^{\alpha}(j)\right)^{2}}}
$$

$$
\mathcal{O}_{k+1, \bar{l}}\left(L^{\alpha}\right)=0 .
$$


Proof. Let us first prove part (b). Let $k>0$. Then, by Proposition 4.31,

$$
\begin{aligned}
\mathcal{O}_{k+1, l}\left(L^{\alpha}\right)=\sum_{L_{\varepsilon}^{\alpha}} \mathcal{O}_{k+1, l}\left(L_{\varepsilon}^{\alpha}\right) \\
=\sum_{L_{\varepsilon}^{\alpha}}\left(\frac{q+q^{-1}}{2}\right)^{l-k} \prod_{j=k+2}^{l} \frac{q-q^{-1}}{1+\frac{c t\left(L_{\varepsilon}^{\alpha}(j-1)\right)}{\varepsilon\left(L_{\varepsilon}^{\alpha}(j)\right) c t\left(L_{\varepsilon}^{\alpha}(j)\right)}} \\
=\sum_{\varepsilon\left(L_{\varepsilon}^{\alpha}(k+1)\right)= \pm 1}\left(\frac{q+q^{-1}}{2}\right)^{l-k} \prod_{j=k+2}^{l}\left(\sum_{\varepsilon\left(L_{\varepsilon}^{\alpha}(j)\right)= \pm 1} \frac{q-q^{-1}}{1+\frac{c t\left(L_{\varepsilon}^{\alpha}(j-1)\right)}{\varepsilon\left(L_{\varepsilon}^{\alpha}(j)\right) c t\left(L_{\varepsilon}^{\alpha}(j)\right)}}\right) \\
=\sum_{\varepsilon\left(L_{\varepsilon}^{\alpha}(k+1)\right)= \pm 1}\left(\frac{q+q^{-1}}{2}\right)^{l-k} \prod_{j=k+2}^{l}\left(\frac{q-q^{-1}}{1+\frac{c t\left(L_{\varepsilon}^{\alpha}(j-1)\right)}{c t\left(L_{\varepsilon}^{\alpha}(j)\right)}}+\frac{q-q^{-1}}{1-\frac{c t\left(L_{\varepsilon}^{\alpha}(j-1)\right)}{c t\left(L_{\varepsilon}^{\alpha}(j)\right)}}\right) \\
=2\left(\frac{q+q^{-1}}{2}\right)^{l-k} \prod_{j=k+2}^{l} \frac{2\left(q-q^{-1}\right)}{1-\frac{c t\left(L_{\varepsilon}^{\alpha}(j-1)\right)^{2}}{c t\left(L_{\varepsilon}^{\alpha}(j)\right)^{2}}} \\
=\left(q+q^{-1}\right) \prod_{j=k+2}^{l} \frac{\left(q^{2}-q^{-2}\right)}{1-\frac{c t\left(L_{\varepsilon}^{\alpha}(j-1)\right)^{2}}{c t\left(L_{\varepsilon}^{\alpha}(j)\right)^{2}}} .
\end{aligned}
$$

The first two equalities in part (a) follows from the first two equalities in part (a) of Proposition 4.31. The third equality in part (a) is the same as case (b) except that $k=0$. It follows by a similar calculation, except that the $\operatorname{sign} \varepsilon\left(L_{\varepsilon}^{\alpha}(1)\right)$ is forced by the condition that the product of the signs in $L_{\varepsilon}^{\alpha}$ is $(-1)^{n / 2-1}$. Thus this case does not have the extra factor of 2 that appears when $k>0$.

For part (c), we have that

$$
\begin{aligned}
\mathcal{O}_{k+1, \bar{l}}\left(L^{\alpha}\right)= & \sum_{\substack{\varepsilon(T(j))= \pm 1 \\
k+1 \leq j \leq l}} \mathcal{O}_{k+1, \bar{l}}\left(L_{\varepsilon}^{\alpha}\right) \\
= & \sum_{\substack{\varepsilon\left(L_{\varepsilon}^{\alpha}(j)\right)= \pm 1 \\
k+1<j \leq l}} \sum_{\varepsilon\left(L_{\varepsilon}^{\alpha}(k+1)\right)= \pm 1}(-1)^{k+1}\left(\prod_{j=2}^{k} \varepsilon\left(L_{\varepsilon}^{\alpha}(j)\right)\right) \varepsilon\left(L_{\varepsilon}^{\alpha}(k+1)\right) \\
& \cdot c t\left(L_{\varepsilon}^{\alpha}(k+1)\right)\left(\frac{q+q^{-1}}{2}\right)^{l-k} \prod_{j=k+2}^{l} \frac{q-q^{-1}}{1+\frac{c t\left(L_{\varepsilon}^{\alpha}(j-1)\right)}{\varepsilon\left(L_{\varepsilon}^{\alpha}(j)\right) c t\left(L_{\varepsilon}^{\alpha}(j)\right)}} \\
= & 0,
\end{aligned}
$$

since $\sum_{\varepsilon\left(L_{\varepsilon}^{\alpha}(k+1)\right)= \pm 1} \varepsilon\left(L_{\varepsilon}^{\alpha}(k+1)\right)=1+(-1)=0$.

Theorem 4.35. Let $\vec{l}=\left(l_{1}, \ldots, l_{t}\right)$ be an increasing sequence. Let $\widetilde{T}_{\vec{l}}$ be the element of $H D_{n}\left(q^{2}\right)$ determined by (4.16)-(4.17) and let $T_{\vec{l}}^{B}$ be the element of $H B_{n}\left(1, q^{2}\right)$ determined by (2.13). When $\vec{l}$ is a marked increasing sequence we ignore the mark when constructing $T_{\vec{l}}^{B}$.

(a) Then, for each pair of partitions $(\alpha, \beta)$ such that $|\alpha|+|\beta|=n$,

$$
\chi_{H D_{n}\left(q^{2}\right)}^{(\alpha, \beta)}\left(\widetilde{T}_{\vec{l}}\right)=\chi_{H B_{n}\left(1, q^{2}\right)}^{(\alpha, \beta)}\left(T_{\vec{l}}^{B}\right) .
$$

(b) Suppose that $n$ is even, and let $m=n / 2$. If the increasing sequence $\vec{l}$ is such that all $l_{i}$ are even and all $l_{i}$ are unbarred, then let $\vec{m}=\left(m_{1}, m_{2}, \ldots, m_{t}\right)$ be the 
increasing sequence given by $2 m_{i}=l_{i}$ for each $1 \leq i \leq t$. Let $T_{\vec{m}}^{A}$ be $T_{\vec{m}}^{B}$ viewed as an element of $H A_{m-1}\left(q^{4}\right)$. Then, for each partition $\alpha$ such that $2|\alpha|=n$,

$$
\begin{aligned}
& \chi_{H D_{n}\left(q^{2}\right)}^{(\alpha, \alpha)^{ \pm}}\left(\widetilde{T}_{\vec{l}}\right) \\
& =\frac{1}{2} \chi_{H B_{n}\left(1, q^{2}\right)}^{(\alpha, \alpha)}\left(T_{\vec{l}}^{B}\right) \pm \begin{cases}\frac{1}{2}\left(q+q^{-1}\right)^{t} \chi_{H A_{m-1}\left(q^{4}\right)}^{\alpha}\left(T_{\vec{m}}^{A}\right), & \text { if all } l_{i} \text { are even, } \\
& \text { all } l_{i} \text { are unbarred, } \\
& \text { and } l_{1} \text { is unmarked } ; \\
-\frac{1}{2}\left(q+q^{-1}\right)^{t} \chi_{H A_{m-1}\left(q^{4}\right)}^{\alpha}\left(T_{\vec{m}}^{A}\right), & \text { if all } l_{i} \text { are even, } \\
& \text { all } l_{i} \text { are unbarred, } \\
& \text { and } l_{1} \text { is marked; } \\
& \text { otherwise, }\end{cases}
\end{aligned}
$$

where $\chi_{H D_{n}\left(q^{2}\right)}^{\lambda}, \chi_{H B_{n}\left(1, q^{2}\right)}^{\lambda}$, and $\chi_{H A_{m-1}\left(q^{4}\right)}^{\lambda}$ denote the irreducible characters of $H D_{n}\left(q^{2}\right), H B_{n}\left(1, q^{2}\right)$, and $H A_{m-1}\left(q^{4}\right)$, respectively, that correspond to the shape $\lambda$.

Proof. Part (a) is a restatement of Theorem 4.21. To prove part (b) let $m=n / 2$. By Proposition 4.27 we have that

$$
\chi_{H D_{n}\left(q^{2}\right)}^{(\alpha, \alpha)^{ \pm}}\left(\widetilde{T}_{\vec{l}}\right)=\left\{\begin{array}{l}
\frac{1}{2} \chi_{H B_{n}\left(1, q^{2}\right)}^{(\alpha, \alpha)}\left(T_{\vec{l}}^{B}\right) \pm \sum_{L \in \mathcal{A} \mathcal{L}_{2}(\alpha, \alpha)} \mathcal{O}_{\vec{l}}(L), \quad \text { if } \vec{l} \text { is even } \\
\frac{1}{2} \chi_{H B_{n}\left(1, q^{2}\right)}^{(\alpha, \alpha)}\left(T_{\vec{l}}^{B}\right), \quad \text { otherwise }
\end{array}\right.
$$

If $\vec{l}=\left(l_{1}, \ldots, l_{t}\right)$ is even, let $\vec{m}=\left(m_{1}, m_{2}, \ldots, m_{t}\right)$ be the increasing sequence given by $m_{i}=l_{i} / 2$, for $1 \leq i \leq t$. Let $m_{1}$ be marked if $l_{1}$ is marked and let $m_{i}$ be barred if $l_{i}$ is barred. Then it follows from definitions of $\mathcal{O}_{k l}\left(L_{\varepsilon}^{\alpha}\right)$ and $\mathcal{O}_{k l}\left(L^{\alpha}\right)$ in Proposition 4.31 and (4.32), respectively, that

$$
\sum_{L \in \mathcal{A} \mathcal{L}_{2}(\alpha, \alpha)} \mathcal{O}_{\vec{l}}(L)=\sum_{L^{\alpha}} \mathcal{O}_{1, m_{1}}\left(L^{\alpha}\right) \mathcal{O}_{\left|m_{1}\right|+1, m_{2}}\left(L^{\alpha}\right) \ldots \mathcal{O}_{\left|m_{t-1}\right|+1, m_{t}}\left(L^{\alpha}\right)
$$

where the last sum is over all (ordinary, unsigned) standard tableaux of shape $\alpha$.

If $\vec{m}$ has any bars, then it necessarily has an even number of bars, and

$$
\sum_{L^{\alpha}} \mathcal{O}_{1, m_{1}}\left(L^{\alpha}\right) \mathcal{O}_{\left|m_{1}\right|+1, m_{2}}\left(L^{\alpha}\right) \ldots \mathcal{O}_{\left|m_{t-1}\right|+1, m_{t}}\left(L^{\alpha}\right)=0
$$

since, by Proposition $4.34, \mathcal{O}_{k, \bar{l}}\left(L^{\alpha}\right)=0$ when $k>1$.

Now assume $\vec{m}=\left(m_{1}, \ldots, m_{t}\right)$ has no bars. Then, by the definition of $\left(g_{k}\right)_{L^{\alpha} L^{\alpha}}$ in (2.5) and by Proposition 4.31, we have

$$
\begin{aligned}
& \mathcal{O}_{1, m}\left(L^{\alpha}\right)=\frac{q+q^{-1}}{2} \Delta_{1, m}\left(L^{\alpha}\right), \\
& \mathcal{O}_{1, \check{m}}\left(L^{\alpha}\right)=-\frac{q+q^{-1}}{2} \delta_{1, m}\left(L^{\alpha}\right), \quad \text { and } \\
& \mathcal{O}_{k, m}\left(L^{\alpha}\right)=\left(q+q^{-1}\right) \Delta_{k, m}\left(L^{\alpha}\right), \quad k>1,
\end{aligned}
$$


where $\Delta_{k, m}\left(L^{\alpha}\right)$ is as defined in (2.16) except with respect to the algebra $H B_{m}\left(1, q^{4}\right)$. Thus, if $\vec{l}=\left(m_{1}, \ldots, m_{t}\right)$ is unmarked (and has no bars), we have

$$
\begin{aligned}
\sum_{L^{\alpha}} \mathcal{O}_{1, m_{1}}\left(L^{\alpha}\right) \mathcal{O}_{\left|m_{1}\right|+1, m_{2}}\left(L^{\alpha}\right) \ldots \mathcal{O}_{\left|m_{t-1}\right|+1, m_{t}}\left(L^{\alpha}\right) \\
=\frac{1}{2}\left(q+q^{-1}\right)^{t} \sum_{L^{\alpha}} \Delta_{1, m_{1}}\left(L^{\alpha}\right) \Delta_{\left|m_{1}\right|+1, m_{2}}\left(L^{\alpha}\right) \ldots \Delta_{\left|m_{t-1}\right|+1, m_{t}}\left(L^{\alpha}\right) \\
=\frac{1}{2}\left(q+q^{-1}\right)^{t} \chi_{H B_{n}\left(1, q^{4}\right)}^{(\alpha, \varnothing)}\left(T_{\vec{m}}^{B}\right) \text { (by Theorem 2.20) } \\
=\frac{1}{2}\left(q+q^{-1}\right)^{t} \chi_{H A_{m-1}\left(q^{4}\right)}^{\alpha}\left(T_{\vec{m}}^{A}\right) \text { (by Theorem 3.4), }
\end{aligned}
$$

where $T_{\vec{m}}^{B}$ is the same element as $T_{\vec{m}}^{A}$ except viewed as an element of $H B_{m}\left(1, q^{4}\right)$.

The case where $\vec{m}$ is marked is entirely similar. The only difference is the sign appearing in $\mathcal{O}_{1, \check{m}_{1}}\left(L^{\alpha}\right)$. The theorem now follows from Proposition 4.27.

\section{Greene's Poset theOREM}

Curtis Greene $[\mathrm{Gr}]$ uses the theory of partially ordered sets (posets) and Möbius functions to prove a rational function identity ( $[\mathrm{Gr}]$, Theorem 3.3) which can be used to derive the Murnaghan-Nakayama rule for symmetric group characters. In this section, we modify Greene's theorem so that it can be applied to computing Murnaghan-Nakayama rules for the irreducible characters of the Iwahori-Hecke algebras of type $A_{n-1}, B_{n}$, and $D_{n}$. All of the results of this section are only slight modifications and generalizations of the results in $[\mathrm{Gr}]$. This section is completely independent of sections 2,3 , and 4 .

Greene's poset theorem holds for posets $P$ which are planar in the (strong) sense that their Hasse diagrams may be order-embedded in $\mathbb{R} \times \mathbb{R}$ without edge crossings even when extra bottom and top elements are added (see $[\mathrm{Gr}]$ for details). An example of a nonplanar poset is shown in Figure 5.1.

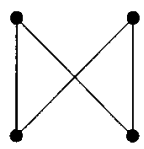

Figure 5.1

The set $[n]=\{1,2, \ldots, n\}$ with its usual order forms a poset with the special property that any two elements are comparable. We call such posets chains. If $P$ is a poset with $n$ elements, then a linear extension of $P$ is a chain $L=\left(P, \leq_{L}\right)$ such that the underlying set is $P$ and such that the relations in $L$ form an extension of the relations in $P$ to a chain. We denote by $\mathcal{L}(P)$ the set of all linear extensions of the poset $P$.

The Möbius function of a poset $P, \mu: P \times P \rightarrow \mathbb{Z}$, is defined inductively for elements $a, b \in P$ by

$$
\mu(a, b)=\mu_{P}(a, b)= \begin{cases}1 & \text { if } a=b, \\ -\sum_{a \leq x<b} \mu(x, z) & \text { if } a<b, \\ 0 & \text { if } a \leq \leq b .\end{cases}
$$

(See [Sta] for more details on Möbius functions.) 
The Main Poset Theorem. Let $\widehat{P}$ be a planar poset with a unique minimal element $u$, and let $x_{a}, a \in \widehat{P}$ be a set of commutative variables indexed by the elements of $\widehat{P}$. Let $q$ be an indeterminate, define

$$
w t(a, b)=\frac{1-x_{a} x_{b}^{-1}}{q-q^{-1}}, \quad \text { for all } a<b \text { in } \widehat{P} \text { with } a \neq u,
$$

and suppose that either

$$
\begin{array}{ccc}
\operatorname{llwt}(u, a) & =1, & \text { or } \\
w t(u, a) & =x_{a}^{-1}, & \text { or } \\
w t(u, a) & =\frac{1-x_{u} x_{a}^{-1}}{\left(q-q^{-1}\right)},
\end{array}
$$

for all $a \in \widehat{P}$ with $a>u$. Define

$$
\kappa(\widehat{P})= \begin{cases}q-q^{-1}, & \text { if } w t(u, a)=1 \text { for all } a \in \widehat{P}, a>u \\ 0, & \text { if } w t(u, a)=x_{a}^{-1} \text { for all } a \in \widehat{P}, a>u \\ 1, & \text { if } w t(u, a)=\frac{1-x_{u} x_{a}^{-1}}{\left(q-q^{-1}\right)} \text { for all } a \in \widehat{P}, a>u\end{cases}
$$

Let $P=\widehat{P}-\{u\}$ be the poset obtained by removing the element $u$ from $\widehat{P}$, and let $c c(\widehat{P})$ be the number of connected components of $P$.

Theorem 5.3. Let $\widehat{P}$ be a planar poset with a unique minimal element $u$, and let $\mu$ be the Möbius function of $\widehat{P}$. With notations as in the previous paragraph, define

$$
\Delta(\widehat{P})=\prod_{\substack{a, b \in \widehat{P} \\ a \neq b}} w t(a, b)^{\mu(a, b)}
$$

Then

$$
\sum_{\widehat{L} \in \mathcal{L}(\widehat{P})} \Delta(\widehat{L})=\kappa(\widehat{P})^{c c(\widehat{P})-1} \Delta(\widehat{P})
$$

where $\mathcal{L}(\widehat{P})$ is the set of linear extensions of $\widehat{P}$.

Proof. The proof is by induction on the size of $\widehat{P}$ and by reverse induction on the number of order relations. Let $|\widehat{P}|=n$, and assume that the theorem holds for all posets $\widehat{Q}$ with $|\widehat{Q}|<n$ and all posets $\widehat{Q}$ with $|\widehat{Q}|=n$ having more order relations than $\widehat{P}$. The base case, when $\widehat{P}$ is a chain, is trivial, since a chain has only one linear extension, itself.

Case 0. Suppose that $P=\widehat{P}-\{u\}$ has a unique minimal element $v$. Then $\operatorname{cc}(\widehat{P})-1=1-1=0$. Since $P$ has fewer elements than $\widehat{P}$, the following holds by induction,

$$
\begin{aligned}
\sum_{\widehat{L} \in \mathcal{L}(\widehat{P})} \Delta(\widehat{L}) & =w t(u, v)^{\mu(u, v)} \sum_{L \in \mathcal{L}(\widehat{P})} \Delta(L) \\
& =w t(u, v)^{\mu(u, v)} \kappa(P)^{c c(P)-1} \Delta(P) .
\end{aligned}
$$


Now note that $\kappa(P)=1$ since $w t(v, a)=\left(1-x_{v} x_{a}^{-1}\right) /\left(q-q^{-1}\right)$ for all $a \in P, a>v$. Thus,

$$
\begin{aligned}
\sum_{\widehat{L} \in \mathcal{L}(\widehat{P})} \Delta(\widehat{L}) & =w t(u, v)^{\mu(u, v)} \Delta(P)=\kappa(\widehat{P})^{0} \Delta(\widehat{P}) \\
& =\kappa(\widehat{P})^{c c(\widehat{P})-1} \Delta(\widehat{P}) .
\end{aligned}
$$

Cases 1 and 2. Suppose that $P=\widehat{P} \backslash\{u\}$ has more than one minimal element. Let $s$ and $t$ be two adjacent minimal elements (in a path along the boundary of $P$ ). Let $\widehat{P}_{A}$ be the same poset as $\widehat{P}$ except add in the extra relation $s \leq t$ and all other relations implied by transitivity. Let $\widehat{P}_{B}$ be the same poset as $\widehat{P}$ except add in the extra relation $t \leq s$ and all other relations implied by transitivity. To summarize,

$$
\begin{aligned}
& \widehat{P}: \text { has unique minimal element } u \\
& \quad \text { and } P=\widehat{P} \backslash\{u\} \text { has adjacent minimal elements } s, t ; \\
& \widehat{P}_{A}: \widehat{P} \text { with the extra relation } s \leq t ; \\
& \widehat{P}_{B}: \widehat{P} \text { with the extra relation } t \leq s .
\end{aligned}
$$

Note that $\widehat{P}_{A}$ and $\widehat{P}_{B}$ are both planar posets with unique minimal element $u$ and that both $\widehat{P}_{A}$ and $\widehat{P}_{B}$ have more relations than $\widehat{P}$. Since $w t$ is the same on $\widehat{P}, \widehat{P}_{A}$, and $\widehat{P}_{B}$, we have that $\kappa(\widehat{P})=\kappa\left(\widehat{P}_{A}\right)=\kappa\left(\widehat{P}_{B}\right)$. Moreover, $\operatorname{cc}\left(\widehat{P}_{A}\right)=\operatorname{cc}\left(\widehat{P}_{B}\right)$. Let $\mu, \mu_{A}$, and $\mu_{B}$ denote the Möbius functions on the posets $\widehat{P}, \widehat{P}_{A}$, and $\widehat{P}_{B}$, respectively. Then, by induction,

$$
\begin{aligned}
& \sum_{L \in \mathcal{L}(\widehat{P})} \Delta(L)=\sum_{L \in \mathcal{L}\left(\widehat{P}_{A}\right)} \Delta(L)+\sum_{L \in \mathcal{L}\left(\widehat{P}_{B}\right)} \Delta(L) \\
& =\kappa\left(\widehat{P}_{A}\right)^{c c\left(\widehat{P}_{A}\right)-1} \prod_{\substack{a, b \in \widehat{P}_{A} \\
a \neq b}} w t(a, b)^{\mu_{A}(a, b)}+\kappa\left(\widehat{P}_{B}\right)^{c c\left(\widehat{P}_{B}\right)-1} \prod_{\substack{a, b \in \widehat{P}_{B} \\
a \neq b}} w t(a, b)^{\mu_{B}(a, b)} \\
& =\kappa\left(\widehat{P}_{A}\right)^{c c\left(\widehat{P}_{A}\right)-1} \prod_{\substack{a, b \in P \\
a \neq b}} w t(a, b)^{\mu(a, b)}\left(\prod_{\substack{a, b \in P_{A} \\
a \neq b}} w t(a, b)^{\mu_{A}(a, b)-\mu(a, b)}+\prod_{\substack{a, b \in P_{B} \\
a \neq b}} w t(a, b)^{\mu_{B}(a, b)-\mu(a, b)}\right) \\
& =\kappa(\widehat{P})^{c c\left(\widehat{P}_{A}\right)-1} \Delta(\widehat{P})\left(\prod_{\substack{a, b \in P_{A} \\
a \neq b}} w t(a, b)^{\mu_{A}(a, b)-\mu(a, b)}\right) \\
& \left.+\prod_{\substack{a, b \in P_{B} \\
a \neq b}} w t(a, b)^{\mu_{B}(a, b)-\mu(a, b)}\right)
\end{aligned}
$$


Case 1. In $\widehat{P}$, the least common multiple $d=s \wedge t$ of $s$ and $t$ does not exist. Then $c c\left(\widehat{P}_{A}\right)=c c(\widehat{P})-1$. In [Gr], C. Greene uses the theory of Möbius algebras to compute the differences

$$
\begin{aligned}
\mu_{A}(u, t)-\mu(u, t) & =1, & & \mu_{B}(u, s)-\mu(u, s)=1, \\
\mu_{A}(s, t)-\mu(s, t) & =-2, & & \mu_{B}(t, s)-\mu(t, s)=-1,
\end{aligned}
$$

and $\mu_{A}(a, b)-\mu(a, b)=\mu_{B}(a, b)-\mu(a, b)=0$ for all other $a \neq b$ in $P$. Thus,

$$
\begin{aligned}
\prod_{s \neq t} w t(s, t)^{\mu_{A}(s, t)-\mu(s, t)}+ & \prod_{s \neq t} w t(s, t)^{\mu_{B}(s, t)-\mu(s, t)}=\frac{w t(u, t)}{w t(s, t)}+\frac{w t(u, s)}{w t(t, s)} \\
= & \left\{\begin{array}{ll}
1, & \text { if } w t(u, a)=\frac{1-x_{u} x_{a}^{-1}}{\left(q-q^{-1}\right)} \\
q-q^{-1}, & \text { if } w t(u, a)=1 ; \text { for all } a>u, \\
0, & \text { if } w t(u, a)=x_{a}^{-1},
\end{array}=\kappa(\widehat{P}) .\right.
\end{aligned}
$$

Case 2. In $\widehat{P}$, the least common multiple $d=s \vee t$ exists. Then $c c\left(\widehat{P}_{A}\right)=c c(\widehat{P})$. In $[\mathrm{Gr}]$, C. Greene explains how to use the theory of Möbius algebras to compute the differences

$$
\begin{aligned}
\mu_{A}(u, t)-\mu(u, t) & =t, & & \mu_{B}(u, s)-\mu(u, s)=1, \\
\mu_{A}(u, d)-\mu(u, d) & =-1, & & \mu_{B}(u, d)-\mu(u, d)=-1, \\
\mu_{A}(s, t)-\mu(s, t) & =-1, & & \mu_{B}(t, s)-\mu(t, s)=-1, \\
\mu_{A}(s, d)-\mu(s, d) & =1, & & \mu_{B}(t, d)-\mu(t, d)=1,
\end{aligned}
$$

and $\mu_{A}(a, b)-\mu(a, b)=\mu_{B}(a, b)-\mu(a, b)=0$, for all other $a \neq b$ in $P$. Thus

$$
\begin{aligned}
\prod_{s \neq t} w t(s, t)^{\mu_{A}(s, t)-\mu(s, t)}+\prod_{s \neq t} w t(s, t)^{\mu_{B}(s, t)-\mu(s, t)} \\
=\frac{w t(s, d) w t(u, t)}{w t(s, t) w t(u, d)}+\frac{w t(t, d) w t(u, s)}{w t(t, s) w t(u, d)} \\
= \begin{cases}1, & \text { if } w t(u, a)=\frac{1-x_{u} x_{a}^{-1}}{\left(q-q^{-1}\right)} ; \\
1, & \text { if } w t(u, a)=1 ; \\
1, & \text { if } w t(u, a)=x_{a}^{-1} ;\end{cases}
\end{aligned}
$$

Shapes and Standard Tableaux. There is a natural extension of the theory of partitions and tableaux to the theory of partially ordered sets in which partitions correspond to posets and tableaux to their linear extensions. (For a full treatment of this subject, see [Sta], whose notation we use here.) If $\rho$ and $\lambda$ are partitions with $\rho \subseteq \lambda$, then we construct a corresponding poset $P_{\lambda / \rho}$ by which has Hasse diagram given by placing a node in each box of $\lambda / \rho$ and drawing edges connecting nodes in adjacent boxes. The order relation in this poset is so that the smallest nodes are in the upper left corners, as shown in Figure 5.5.

Note that posets corresponding to skew-shapes are always planar.

If $s$ is a minimal element of the poset $P_{\lambda / \rho}$ then we say that the corresponding box in the shape $\lambda / \rho$ is a sharp corner. If $d$ is an element of the poset $P_{\lambda / \rho}$ such that $d=s \vee t$ where $s$ and $t$ are two adjacent sharp corners of $\lambda / \rho$, then we say that 


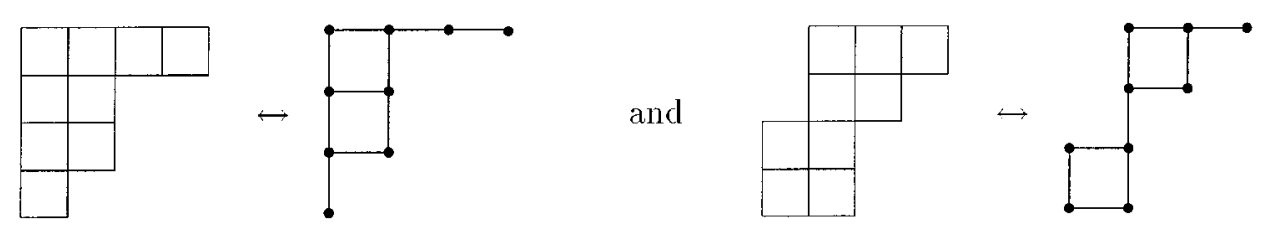

FigURE 5.5

the box of $\lambda / \rho$ corresponding to $d$ is a dull corner. In Figure 2.21 the sharp and dull corners of the partition are exactly the sharp and dull corners of the corresponding poset.

Let $\widehat{P}_{\lambda / \rho}$ be the poset $P_{\lambda / \rho} \cup\{u\}$ where the adjoined element $u$ satisfies $u \leq a$ for all $a \in P_{\lambda / \rho}$. Let $\mu$ be the Möbius function of the poset $\widehat{P}_{\lambda / \rho}$. Intervals of $\widehat{P}_{\lambda / \rho}$ of the form of Figure 5.6.

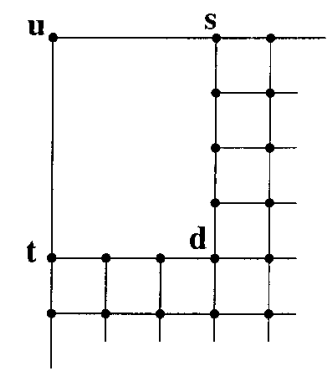

Figure 5.6

where $d=s \vee t$, have $\mu(u, s)=\mu(u, t)=-1, \mu(u, d)=1$, and $\mu(u, x)=0$ for all other $u \leq x$. Intervals of $\widehat{P}_{\lambda / \rho}$ of the form are given in Figure 5.7.

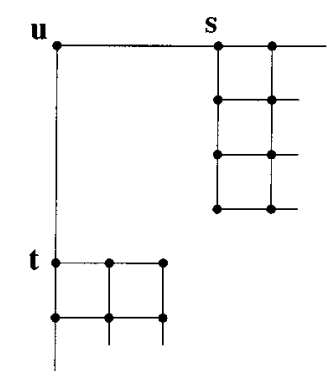

FiguRE 5.7

where $s \vee t$ does not exist, have $\mu(u, s)=\mu(u, t)=-1$, and $\mu(u, x)=0$ for all other $u \leq x$.

Theorem 5.8. Let $\lambda$ be any shape (or skew shape) with $n$ boxes and let cc be the number of connected components of $\lambda$. Let $\left\{x_{b}\right\}$ be a set of commutative variables indexed by the boxes $b \in \lambda$ and let $q$ be an indeterminate. For each standard tableau 
$L$ of shape $\lambda$ and each $1 \leq i \leq n$, let $L(i)$ denote the box of $L$ containing $i$, and define

$$
\Delta(L)=\prod_{i=2}^{n} \frac{\left(q-q^{-1}\right)}{1-x_{L(i)} x_{L(i-1)}^{-1}}, \quad \text { and } \quad \bar{\Delta}(L)=x_{L(1)} \prod_{i=2}^{n} \frac{\left(q-q^{-1}\right)}{1-x_{L(i)} x_{L(i-1)}^{-1}} .
$$

Then

$$
\sum_{L \in \mathcal{L}(\lambda)} \Delta(L)=\left(q-q^{-1}\right)^{c c-1}\left(\frac{\prod_{D}\left(1-x_{b} x_{a}^{-1}\right)}{\prod_{R}\left(1-x_{b} x_{a}^{-1}\right) \prod_{C}\left(1-x_{b} x_{a}^{-1}\right)}\right),
$$

and

$$
\sum_{L \in \mathcal{L}(\lambda)} \bar{\Delta}(L)=\left\{\begin{array}{l}
\frac{\prod_{s \in S C} x_{s}}{\prod_{d \in D C} x_{d}}\left(\frac{\prod_{D}\left(1-x_{b} x_{a}^{-1}\right)}{\prod_{R}\left(1-x_{b} x_{a}^{-1}\right) \prod_{C}\left(1-x_{b} x_{a}^{-1}\right)}\right) \\
0, \quad \text { otherwise }
\end{array}\right.
$$

where

$D$ is the set of pairs $(a, b)$ of boxes in $\lambda$ adjacent (northwest to southeast) in a diagonal,

$R$ is the set of pairs $(a, b)$ of boxes in $\lambda$ adjacent (west to east) in a row,

$C$ is the set of pairs $(a, b)$ of boxes in $\lambda$ adjacent (north to south) in a column, $S C$ is the set of sharp corners of $\lambda$, and

$D C$ is the set of dull corners of $\lambda$.

Proof. Let $\lambda$ be a shape (or a skew shape) and let $\mathcal{L}(\lambda)$ be the set of standard tableaux of shape $\lambda$. Linear extensions of the poset $P_{\lambda}$ are in one-to-one correspondence with standard tableaux having skew shape $\lambda$ as follows: Given a standard tableau $T$ of shape $\lambda$ let $T(k)$ denote the box containing $k$ in $T$. Then the standard tableau $T$ corresponds to the linear extension $L$ of the poset $P_{\lambda}$ which has underlying set $P_{\lambda}$ and order relations given by $T(k) \leq_{L} T(l)$ if $k \leq l$. We can identify the standard tableau $T$ with the chain $L$.

Let $\widehat{P}_{\lambda}$ be the poset $P_{\lambda} \cup\{u\}$ where the adjoined element $u$ satisfies $u \leq a$ for all $a \in P_{\lambda}$. The linear extensions of the poset $\widehat{P}_{\lambda}$ are in one-to-one correspondence with the linear extensions of the poset $P_{\lambda}$. Thus, we can identify a standard tableau $T$ of shape $\lambda$ with a linear extension $\widehat{L}$ of the poset $\widehat{P}_{\lambda}$.

Let $\mu$ be the Möbius function of the poset $\widehat{P}_{\lambda}$. The values of the Möbius function of the poset $\widehat{P}_{\lambda}$ are as indicated in (5.6) and (5.7). In particular, if $u$ is the minimal element of $\widehat{P}_{\lambda}$, then it follows from (5.6) that the Möbius function of $\widehat{P}_{\lambda}$ satisfies

$$
\begin{array}{ll}
\mu(u, s)=-1, & \text { if } s \text { is a sharp corner of } \lambda, \\
\mu(u, d)=1, & \text { if } d \text { is a dull corner of } \lambda .
\end{array}
$$

Define weight functions $w t$ and $\overline{w t}$ on $\widehat{P}_{\lambda}$ by

$$
w t(a, b)=\overline{w t}(a, b)=\frac{1-x_{a} x_{b}^{-1}}{q-q^{-1}}, \quad \text { for } a, b \in P_{\lambda}, a \neq b,
$$

and

$$
w t(u, a)=1, \quad \text { and } \quad \overline{w t}(u, a)=x_{a}^{-1}, \quad \text { for } a \in P_{\lambda} .
$$


Then, if $L$ is a standard tableau of shape $\lambda$, which corresponds to a linear extension $\widehat{L}$ of the poset $\widehat{P}_{\lambda}$, we have that

$$
\begin{aligned}
& \Delta(\widehat{L})=\prod_{\substack{a, b \in \widehat{P}_{\lambda} \\
a \neq b}} w t(a, b)^{\mu(a, b)}=\Delta(L) \quad \text { and } \\
& \bar{\Delta}(\widehat{L})=\prod_{\substack{a, b \in \widehat{P}_{\lambda} \\
a \neq b}} \overline{w t}(a, b)^{\mu(a, b)}=\bar{\Delta}(L)
\end{aligned}
$$

where $\Delta(L)$ and $\bar{\Delta}(L)$ are as defined in the statement of the theorem. Thus, we may use Theorem 5.3 to compute

$$
\sum_{L \in \mathcal{L}(\lambda)} \Delta(L) \quad \text { and } \sum_{L \in \mathcal{L}(\lambda)} \bar{\Delta}(L)
$$

The result then follows from the computation of the values of the Möbius function of $\widehat{P}_{\lambda}$ in (5.6), (5.7) and (5.9).

\section{REFERENCES}

[AK] S. Ariki and K. Koike, A Hecke algebra of $(\mathbb{Z} / r \mathbb{Z})$ < $\mathcal{S}_{n}$ and construction of its irreducible representations, Adv. in Math. 106 (1994), 216-243. MR 95h:20006

[ATY] S. Ariki, T. Terasoma, and H. Yamada, Schur-Weyl reciprocity for the Hecke algebra of $(\mathbb{Z} / r \mathbb{Z})$ 乙 $\mathcal{S}_{n}$, preprint 1994.

[CK1] C. J. Cummins and R. C. King, Frobenius formula for characters of Hecke algebras of type $A_{n-1}$, Comm. Algebra 21 (12) (1993), 4397-4423. MR 95b:20011

[CK2] — An algorithm for calculating characters of Hecke algebras $H_{n}(q)$ of type $A_{n-1}$ when $q$ is a root of unity, Comm. Algebra 21 (12) (1993), 4425-4437. MR 95b:20012

[Ge] M. Geck, On the character values of Iwahori-Hecke algebras of exceptional type, Proc. London Math. Soc. (3) 68 (1994), 51-76. MR 94i:20073

[GP] M. Geck and G. Pfeiffer, On the irreducible characters of Hecke algebras, Adv. in Math. 102 (1993), 79-94. MR 94m:20018

[Gr] C. Greene, A rational function identity related to the Murnaghan-Nakayama formula for the characters of $\mathcal{S}_{n}$, J. Alg. Comb. 1 (1992), 235-255. MR 93k:20025

$[\mathrm{H}] \quad$ P. N. Hoefsmit, Representations of Hecke algebras of finite groups with BN-pairs of classical type, Thesis, Univ. of British Columbia, 1974.

[KW] R. C. King and B. G. Wybourne, Representations and traces of the Hecke algebras $H_{n}(q)$ of type $A_{n-1}$, J. Math. Phys. 33 (1992), 4-14. MR 93c:20028

[LR] R. Leduc and A. Ram, A ribbon Hopf algebra approach to the irreducible representations of centralizer algebras, The Brauer, Birman-Wenzl, and Type A Iwahori-Hecke algebras, Adv. in Math. (to appear).

[Mac] I. G. Macdonald, Symmetric functions and Hall polynomials, Oxford Univ. Press, 1979. MR 84g:05003

[P] G. Pfeiffer, Young characters on Coxeter basis elements of Iwahori-Hecke algebras and a Murnaghan-Nakayama formula, J. Algebra 168 (1994), 525-535. MR 95g:20012

[R] A. Ram, A Frobenius formula for the characters of the Hecke algebras, Invent. Math. 106 (1991), 461-488. MR 93c:20029

[Sta] R. Stanley, Enumerative combinatorics, vol. I, Wadsworth \& Brooks/Cole, 1986. MR

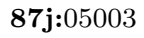

[Ste] J. Stembridge, unpublished notes, 1987. 
[vdJ] J. van der Jeugt, An algorithm for characters of Hecke algebras $H_{n}(q)$ of type $A_{n-1}$, J. Phys. A 24 (1991), 3719-3724. MR 91i:05224

[Y] A. Young, Quantitative substitutional analysis I-IX, Proc. London Math. Soc. (1901-1952). MR 14:126e

Department of Mathematics, Macalester College, St. Paul, Minnesota 55105

E-mail address: halverson@macalstr.edu

Department of Mathematics, University of Wisconsin, Madison, Wisconsin 53706

Current address: Department of Mathematics, Princeton University, Princeton, New Jersey 08544 